\title{
Non-perturbative comparison of QCD effective charges
}

\author{
A. C. Aguilar, ${ }^{1}$ D. Binosi, ${ }^{2}$ J. Papavassiliou, ${ }^{3}$ and J. Rodríguez-Quintero ${ }^{4}$ \\ ${ }^{1}$ Federal University of $A B C, C C N H$, Rua Santa Adélia 166, \\ CEP 09210-170, Santo André, Brazil. \\ ${ }^{2}$ European Centre for Theoretical Studies in Nuclear Physics and Related Areas (ECT*), \\ Villa Tambosi, Strada delle Tabarelle 286, I-38050 Villazzano (TN), Italy. \\ ${ }^{3}$ Department of Theoretical Physics and IFIC, \\ University of Valencia-CSIC, E-46100, Valencia, Spain. \\ ${ }^{4}$ Department of Applied Physics, University of Huelva, E-21071 Huelva, Spain.
}

\begin{abstract}
We study the non-perturbative behavior of two versions of the QCD effective charge, one obtained from the pinch technique gluon self-energy, and one from the ghost-gluon vertex. Despite their distinct theoretical origin, due to a fundamental identity relating various of the ingredients appearing in their respective definitions, the two effective charges are almost identical in the entire range of physical momenta, and coincide exactly in the deep infrared, where they freeze at a common finite value. Specifically, the dressing function of the ghost propagator is related to the two form factors in the Lorentz decomposition of a certain Green's function, appearing in a variety of field-theoretic contexts. The central identity, which is valid only in the Landau gauge, is derived from the Schwinger-Dyson equations governing the dynamics of the aforementioned quantities. The renormalization procedure that preserves the validity of the identity is carried out, and various relevant kinematic limits and physically motivated approximations are studied in detail. A crucial ingredient in this analysis is the infrared finiteness of the gluon propagator, which is inextricably connected with the aforementioned freezing of the effective charges. Some important issues related to the consistent definition of the effective charge in the presence of such a gluon propagator are resolved. We finally present a detailed numerical study of a special set of Schwinger-Dyson equations, whose solutions determine the non-perturbative dynamics of the quantities composing the two effective charges.
\end{abstract}

PACS numbers: 11.15.Tk 12.38.Lg, 12.38.Aw, 


\section{INTRODUCTION}

The infrared behavior of the QCD effective charge is of considerable theoretical and phenomenological interest [1, 22, 3, 4]. This quantity, when correctly defined, provides a continuous interpolation between two physically distinct regimes: the deep ultraviolet (UV), where perturbation theory works well, and the deep infrared (IR), where non-perturbative techniques must be employed. In fact, the effective charge is intimately connected with two phenomena that are of central importance to QCD: asymptotic freedom in the UV, and dynamical gluon mass generation in the IR [1, 5]. Thus, while perturbatively it captures asymptotic freedom, it also exposes, due to the appearance of the Landau pole, the need of a non-perturbative regulating mechanism. Therefore,its low-energy behavior conveys valuable information about the way the theory cures the IR instabilities, namely through the nonperturbative generation of a dynamical mass scale, which tames the perturbative Landau pole. What makes the effective charge such an interesting quantity to study is its strong dependence on the detailed characteristics of some of the most fundamental Green's functions of QCD, such as the gluon and ghost propagators. Indeed, the basic ingredients that enter in its definition must contain the right information and be combined in a very precise way in order to endow the effective charge with the required physical and field-theoretic properties.

In this article we will focus on two characteristic definitions of the effective charge, frequently employed in the literature. The first definition is obtained within the pinch technique (PT) framework [1, 4, 6], and its correspondence [7, 8] with the background-field method

(BFM) [9]. The PT effective charge, to be denoted by $\alpha_{\mathrm{PT}}\left(q^{2}\right)$, constitutes the most direct non-abelian generalization of the familiar concept of the QED effective charge. The second definition of the QCD effective charge, to be denoted by $\alpha_{\mathrm{gh}}\left(q^{2}\right)$, involves the ghost and gluon self-energies, in the Landau gauge, and in the kinematic configuration where the well-known Taylor non-renormalization theorem [10, 11] becomes applicable. $\alpha_{\mathrm{gh}}\left(q^{2}\right)$ has been employed extensively in lattice studies (see for instance [12, 13] and references therein), where the Landau gauge is the standard choice for the simulation of the gluon and ghost propagators, as well as in various investigations based on Schwinger-Dyson equations (SDEs) [14, 15]. Even though the theoretical origin of the two aforementioned effective charges is rather distinct, it turns out that, quite remarkably, by virtue of a powerful non-perturbative identity, they are almost identical in the entire range of physical (euclidean) momenta. In fact, most in- 
terestingly, they are exactly equal in the deep IR (i.e., at vanishing momentum transfer, $\left.q^{2}=0\right)$.

As we will see shortly, in the definition of the two effective charges appears a common ingredient, namely the gluon propagator (in the Landau gauge), and two ingredients that are not common. These two non-common ingredients are, a-priori, not related to each other; the role of the aforementioned identity is to furnish a non-trivial connection between them. Specifically, it relates the dressing function of the ghost propagator, denoted by $F\left(q^{2}\right)$, entering into the definition of $\alpha_{\mathrm{gh}}\left(q^{2}\right)$, with a certain function, denoted by $G\left(q^{2}\right)$, appearing in the definition of $\alpha_{\mathrm{PT}}\left(q^{2}\right)$. The function $G\left(q^{2}\right)$ is the form-factor multiplying $g_{\mu \nu}$ in the Lorentz decomposition of a special Green's function, denoted by $\Lambda_{\mu \nu}(q)$, which appears in a variety of field-theoretic contexts. Most notably, $\Lambda_{\mu \nu}(q)$ enters in all "backgroundquantum" identities, i.e. the infinite tower of non-trivial relations connecting the BFM Green's functions to the conventional ones [16, 17]. Notice also that $G\left(q^{2}\right)$ plays a central role in the new SDEs derived within the PT framework [18]; due to the special properties of the Green's functions involved, these new SDEs can be truncated in a manifestly gauge invariant way [5]. The identity in question connects the two non-common ingredients of the two charges, $F\left(q^{2}\right)$ and $G\left(q^{2}\right)$, to the second form factor of $\Lambda_{\mu \nu}(q)$, denoted by $L\left(q^{2}\right)$, in the way shown in Eq. (3.4).

To the best of our knowledge, the identity of Eq. (3.4) was first derived in [19], in connection with the so-called Kugo-Ojima confinement criterion [20]. The same identity was proved in [21], where the general algebraic properties of $S U(N)$ Yang Mills theories in the background Landau gauge were studied; however, no connection with the conventional $R_{\xi}$ Landau gauge was established. More recently, it was revisited in [22], where a new relation between the Kugo-Ojima parameter and the Gribov-Zwanziger horizon function has been advocated. However, to date, the dynamical equations for the quantities appearing in this identity remain largely unknown.

In the present work we derive the central identity starting from the SDEs that govern the dynamics of the relevant functions, namely $F\left(q^{2}\right), G\left(q^{2}\right)$, and $L\left(q^{2}\right)$. These SDEs allow for a detailed study of the individual properties of these three functions, both perturbatively and non-perturbatively. Most importantly, they expose the way these functions depend on the gluon propagator, and furnish a self-consistent framework for studying how an IR finite gluon propagator affects their IR properties. These properties, in turn, are responsible for 
the mild discrepancy between the two effective charges mentioned above.

The paper is organized as follows. In Section II, after introducing the necessary notation and definitions, we outline the basic theoretical ingredients entering into the construction of the two (dimensionful) renormalization-group $(\mathrm{RG})$ invariant quantities, from which the two (dimensionless) effective charges, $\alpha_{\mathrm{PT}}\left(q^{2}\right)$ and $\alpha_{\mathrm{gh}}\left(q^{2}\right)$ will be extracted. Then, we focus on the timely question of how to identify the correct non-perturbative scale in the presence of an IR finite gluon propagator. The central identity of the paper is derived in Section III, starting from the defining SDEs. The renormalization procedure that preserves the validity of the identity is carried out, and various properties are studied in the UV and IR kinematic limits; most notably, we establish that if the gluon propagator is IR finite, then $L(0)=0$. The implications of the identity on the two effective charges are discussed, and a relation between them is established, which is valid for the entire range of euclidean momenta. A detailed numerical analysis and comparison of the two effective charges at different renormalization scales is carried out in Section IV, using as an input the non-perturbative solutions of the SDEs corresponding to the various functions appearing in their definition. Finally, in Section $[\mathrm{V}$ we present our conclusions.

\section{TWO NON-PERTURBATIVE EFFECTIVE CHARGES}

In this section we will first introduce some of the basic filed-theoretic ingredients necessary for the definition of the two effective charges we want to study. Then, we will briefly outline the basic construction and the assumptions involved in the definition of either charge. Finally, we will discuss in detail the important issue of how to extend the two definitions to the non-perturbative regime, and, in particular, the identification of the correct scale in the presence of an IR-finite gluon propagator.

\section{A. Definitions and ingredients}

Let us first introduce the notation and define some of the basic quantities entering into the problem under study.

In the covariant renormalizable $\left(R_{\xi}\right)$ gauges, the gluon propagator $\Delta_{\mu \nu}(q)$ has the form

$$
\Delta_{\mu \nu}(q)=-i\left[P_{\mu \nu}(q) \Delta\left(q^{2}\right)+\xi \frac{q_{\mu} q_{\nu}}{q^{4}}\right],
$$




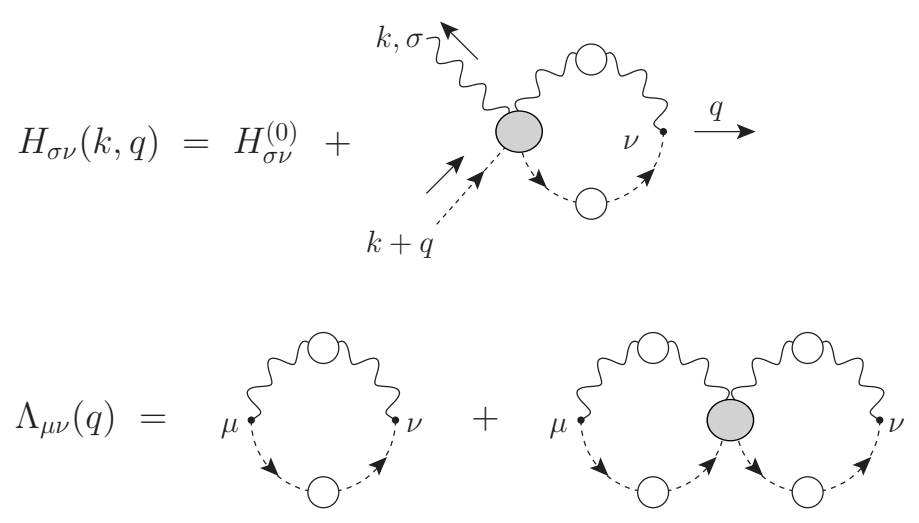

FIG. 1: Diagrammatic representation of the functions $H$ and $\Lambda$.

where $\xi$ denotes the gauge-fixing parameter, and $P_{\mu \nu}(q)=g_{\mu \nu}-q_{\mu} q_{\nu} / q^{2}$ is the usual transverse projector. Finally, $\Delta^{-1}\left(q^{2}\right)=q^{2}+i \Pi\left(q^{2}\right)$, with $\Pi_{\mu \nu}(q)=P_{\mu \nu}(q) \Pi\left(q^{2}\right)$ the gluon self-energy (notice that since $\Pi\left(q^{2}\right)$ has been defined with the imaginary factor $i$ pulled out in front, it is simply given by the corresponding Feynman diagrams in Minkowski space). In addition, the full ghost propagator $D\left(q^{2}\right)$ and its dressing function $F\left(q^{2}\right)$ are related by

$$
D\left(q^{2}\right)=\frac{i F\left(q^{2}\right)}{q^{2}} .
$$

Moreover, the all-order ghost vertex (after factoring out the color structure and the coupling constant $g$ ) will be denoted by $\Gamma_{\mu}(k, q)$ with $k$ representing the momentum of the gluon and $q$ the one of the anti-ghost. The tensorial structure is given by

$$
-\Gamma_{\mu}(k, q)=B_{1}(k, q) q_{\mu}+B_{2}(k, q) k_{\mu}
$$

Thus, at tree-level $\Gamma_{\mu}^{(0)}(k, q)=\Gamma_{\mu}(k, q)=-q_{\mu}$.

An important ingredient for what follows is the two-point function $\Lambda_{\mu \nu}(q)$ represented in Fig. 1, defined by

$$
\begin{aligned}
\Lambda_{\mu \nu}(q) & =-i g^{2} C_{A} \int_{k} H_{\mu \rho}^{(0)} D(k+q) \Delta^{\rho \sigma}(k) H_{\sigma \nu}(k, q), \\
& =g_{\mu \nu} G\left(q^{2}\right)+\frac{q_{\mu} q_{\nu}}{q^{2}} L\left(q^{2}\right),
\end{aligned}
$$

where $C_{\mathrm{A}}$ the Casimir eigenvalue of the adjoint representation $\left[C_{\mathrm{A}}=N\right.$ for $\left.S U(N)\right]$, and $\int_{k} \equiv \mu^{2 \varepsilon}(2 \pi)^{-d} \int d^{d} k$, with $d=4-\epsilon$ the dimension of space-time. The scalar function $G\left(q^{2}\right)$ appearing in the equation above allows the connection between the conventional and BFMPT gluon propagators, and is known to play a central role in the PT formulation of the SDE. 
The function $H_{\mu \nu}(k, q)$ (see Fig. 1 for a diagrammatic definition) is in fact a familiar object [11]: it appears in the all-order Slavnov-Taylor identity satisfied by the standard three-gluon vertex, and is related to the full gluon-ghost vertex by

$$
q^{\nu} H_{\mu \nu}(k, q)=-i \boldsymbol{\Gamma}_{\mu}(k, q) .
$$

At tree-level, $H_{\mu \nu}^{(0)}=i g_{\mu \nu}$. Finally, using the most general Lorentz decomposition of $H_{\mu \nu}$,

$$
-i H_{\mu \nu}(k, q)=A_{1}(k, q) g_{\mu \nu}+A_{2}(k, q) q_{\mu} q_{\nu}+A_{3}(k, q) k_{\mu} k_{\nu}+A_{4}(k, q) q_{\mu} k_{\nu}+A_{5}(k, q) k_{\mu} q_{\nu},
$$

we obtain from (2.3) and (2.5) two constrains for the various form-factors, namely

$$
\begin{aligned}
& B_{1}(k, q)=A_{1}(k, q)+q^{2} A_{2}(k, q)+(k \cdot q) A_{4}(k, q), \\
& B_{2}(k, q)=(k \cdot q) A_{3}(k, q)+q^{2} A_{5}(k, q) .
\end{aligned}
$$

\section{B. The pinch technique effective charge}

The QCD effective charges constructed within the PT uses QED as the basic reference point [23]. In QED, one begins by considering the unrenormalized photon selfenergy $\Pi_{\alpha \beta}^{0}(q)=q^{2} P_{\alpha \beta}(q) \Pi^{0}\left(q^{2}\right)$, where $P_{\alpha \beta}(q)=g_{\alpha \beta}-q_{\alpha} q_{\beta} / q^{2}$ and $\Pi^{0}\left(q^{2}\right)$ is a gaugeindependent function to all orders in perturbation theory. After Dyson summation, we obtain the (process independent) dressed photon propagator between conserved external currents $\Delta_{\alpha \beta}^{0}(q)=\left(g_{\alpha \beta} / q^{2}\right) \Delta^{0}\left(q^{2}\right)$, with $\Delta^{0}\left(q^{2}\right)=-i\left[1+i \Pi^{0}\left(q^{2}\right)\right]^{-1}$. The renormalization procedure introduces the standard relations between renormalized and unrenormalized parameters: $e=Z_{e}^{-1} e_{0}=Z_{f} Z_{A}^{1 / 2} Z_{1}^{-1} e_{0}$ and $1+i \Pi\left(q^{2}\right)=Z_{A}\left[1+i \Pi^{0}\left(q^{2}\right)\right]$, where $Z_{A}\left(Z_{f}\right)$ is the wave-function renormalization constants of the photon (fermion), $Z_{1}$ the vertex renormalization, and $Z_{e}$ is the charge renormalization constant. The Abelian gauge symmetry of the theory gives rise to the fundamental Ward identity (WI) $q^{\alpha} \Gamma_{\alpha}^{0}(p, p+q)=S_{0}^{-1}(p+q)-S_{\mathrm{o}}^{-1}(p)$, where $\Gamma_{\alpha}^{0}$ and $S_{0}(k)$ are the unrenormalized all orders photon-electron vertex and electron propagator, respectively. The requirement that the renormalized vertex $\Gamma_{\alpha}=Z_{1} \Gamma_{\alpha}^{0}$ and the renormalized self-energy $S=Z_{f}^{-1} S_{0}$ satisfy the same identity, implies $Z_{1}=Z_{f}$, from which immediately follows that $Z_{e}=Z_{A}^{-1 / 2}$. Given these relations between the renormalization constants, and after pulling out the trivial factor $g_{\alpha \beta} / q^{2}$, we can form the renormalization group invariant combination, known as the effective charge,

$$
\alpha\left(q^{2}\right)=\frac{e_{0}^{2}}{4 \pi} \Delta^{0}\left(q^{2}\right)=\frac{e^{2}}{4 \pi} \Delta\left(q^{2}\right) .
$$


In QCD, the crucial equality $Z_{1}=Z_{f}$ does not hold, because the WIs are replaced by the more complicated Slavnov-Taylor identities (STIs), involving ghost Green's functions $[11,23]$. Furthermore, the gluon self-energy depends on the gauge-fixing parameter, already at one-loop order. These facts render the QCD generalization of a QED-like effective charge more complicated; however, the theoretical framework of the PT makes this definition possible [1, 6]. The PT rearranges the conventional gauge dependent $n$-point Green's functions, to construct individually gauge independent Green's functions, which, in addition, obey naive (ghost free) WIs . One important point, explained in detail in the literature, is the (all-order) correspondence between the PT and the Feynman gauge of the BFM [7, 8]. In fact, using the methodology introduced in 24], one can generalize the PT construction in such a way as to reach diagrammatically any value of the gauge fixing parameter of the BFM, and in particular the Landau gauge. In what follows we employ the aforementioned generalization of the PT, given that the identity we will eventually derive is valid only in the Landau gauge.

The PT definition of the effective charge relies on the construction of an universal (i.e., process-independent) effective gluon propagator, which captures the running of the QCD $\beta$ function, exactly as happens with the vacuum polarization in the case of QED (See Fig. 22). To fix the ideas, the PT one-loop gluon self-energy reads

$$
\widehat{\Delta}^{-1}\left(q^{2}\right)=q^{2}\left[1+b g^{2} \ln \left(\frac{q^{2}}{\mu^{2}}\right)\right],
$$

where $b=11 C_{A} / 48 \pi^{2}$ is the first coefficient of the QCD $\beta$-function. Due to the Abelian WIs satisfied by the PT effective Green's functions, the new propagator-like quantity $\widehat{\Delta}^{-1}\left(q^{2}\right)$ absorbs all the RG-logs, exactly as happens in QED with the photon self-energy. Then, the renormalization constants of the gauge-coupling and of the PT gluon self-energy, defined as

$$
\begin{aligned}
g\left(\mu^{2}\right) & =Z_{g}^{-1}\left(\mu^{2}\right) g_{0}, \\
\widehat{\Delta}\left(q^{2}, \mu^{2}\right) & =\widehat{Z}_{A}^{-1}\left(\mu^{2}\right) \widehat{\Delta}_{0}\left(q^{2}\right),
\end{aligned}
$$

where the "0" subscript indicates bare quantities, satisfy the QED-like relation

$$
Z_{g}=\widehat{Z}_{A}^{-1 / 2}
$$

Of course, $Z_{g}$ must be obtained under a given renormalization prescription, and the PT gluon self-energy will be then renormalized imposing (2.11). Thus, regardless of the renor- 


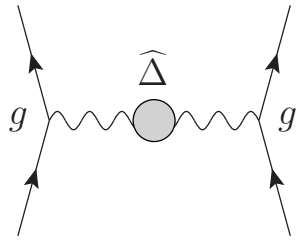

(a)

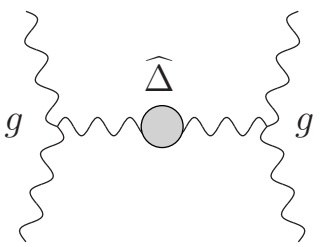

(b)

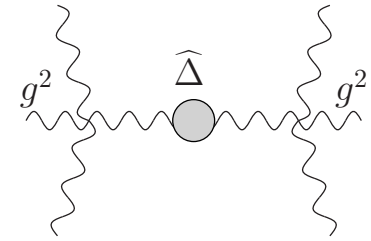

(c)

FIG. 2: The universal PT coupling.

malization prescription chosen, the product

$$
\widehat{d}_{0}\left(q^{2}\right)=g_{0}^{2} \widehat{\Delta}_{0}\left(q^{2}\right)=g^{2}\left(\mu^{2}\right) \widehat{\Delta}\left(q^{2}, \mu^{2}\right)=\widehat{d}\left(q^{2}\right)
$$

retains the same form before and after renormalization, i.e., it forms a RG-invariant $(\mu$ independent) quantity [1].

For asymptotically large momenta one may extract from $\widehat{d}\left(q^{2}\right)$ a dimensionless quantity by writing,

$$
\widehat{d}\left(q^{2}\right)=\frac{\bar{g}^{2}\left(q^{2}\right)}{q^{2}},
$$

where $\bar{g}^{2}\left(q^{2}\right)$ is the RG-invariant effective charge of QCD; at one-loop

$$
\bar{g}^{2}\left(q^{2}\right)=\frac{g^{2}}{1+b g^{2} \ln \left(q^{2} / \mu^{2}\right)}=\frac{1}{b \ln \left(q^{2} / \Lambda_{\mathrm{QCD}}^{2}\right)} .
$$

where $\Lambda_{\mathrm{QCD}}$ denotes an RG-invariant mass scale of a few hundred $\mathrm{MeV}$.

Eq. (2.12) is a non-perturbative relation; therefore it can serve unaltered as the starting point for extracting a non-perturbative effective charge, provided that one has information on the IR behavior of the PT-BFM gluon propagator $\widehat{\Delta}\left(q^{2}\right)$. Interestingly enough, nonperturbative information on the conventional gluon propagator $\Delta\left(q^{2}\right)$ may also be used, by virtue of a general relation connecting $\Delta\left(q^{2}\right)$ and $\widehat{\Delta}\left(q^{2}\right)$. Specifically, a formal all-order relation known as "background-quantum" identity [16, 17] states that

$$
\Delta\left(q^{2}\right)=\left[1+G\left(q^{2}\right)\right]^{2} \widehat{\Delta}\left(q^{2}\right)
$$

Note that, due to its BRST origin, the above relation must be preserved after renormalization. Specifically, denoting by $Z_{\Lambda}$ the (yet unspecified) renormalization constant relating the bare and renormalized functions, $\Lambda_{0}^{\mu \nu}$ and $\Lambda^{\mu \nu}$, through

$$
\Lambda^{\mu \nu}\left(q, \mu^{2}\right)=Z_{\Lambda}\left(\mu^{2}\right) \Lambda_{0}^{\mu \nu}(q)
$$


then from Eqs. (2.15) and (2.11) follows the additional relation

$$
Z_{g}^{-1}=Z_{A}^{1 / 2} Z_{\Lambda}
$$

which is useful for the comparison with the coupling discussed in the following subsection.

It is now easy to verify, at lowest order, that the $1+G\left(q^{2}\right)$ obtained from Eq. (2.4) restores the $\beta$ function coefficient in front of UV logarithm. In that limit [25]

$$
\begin{aligned}
1+G\left(q^{2}\right) & =1+\frac{9}{4} \frac{C_{\mathrm{A}} g^{2}}{48 \pi^{2}} \ln \left(\frac{q^{2}}{\mu^{2}}\right), \\
\Delta^{-1}\left(q^{2}\right) & =q^{2}\left[1+\frac{13}{2} \frac{C_{\mathrm{A}} g^{2}}{48 \pi^{2}} \ln \left(\frac{q^{2}}{\mu^{2}}\right)\right] .
\end{aligned}
$$

Using Eq. (2.15) we therefore recover the $\widehat{\Delta}^{-1}\left(q^{2}\right)$ of Eq. (2.9), as we should.

Then, non-perturbatively, one substitutes into Eq. (2.15) the $1+G\left(q^{2}\right)$ and $\Delta\left(q^{2}\right)$ obtained from either the lattice or SD analysis, to obtain $\widehat{\Delta}\left(q^{2}\right)$. This latter quantity is the non-perturbative generalization of Eq. (2.9); for the same reasons explained above, the combination

$$
\widehat{d}\left(q^{2}\right)=\frac{g^{2} \Delta\left(q^{2}\right)}{\left[1+G\left(q^{2}\right)\right]^{2}},
$$

is an RG-invariant quantity.

\section{The effective charge from the ghost-gluon vertex}

In the previous subsection it has become clear that the PT construction involves a particular combination of two point functions only, with no explicit reference to any of the full vertices of the theory. Thus, as happens in QED, the effective charge so obtained is universal (i.e., it does not depend on the details of the process where the PT propagator is embedded), and depends naturally on a single scale, namely the physical momentum exchange of a given process.

In principle, a definition for the QCD effective charge can be obtained starting from the various QCD vertices ${ }^{1}$, i.e., the ghost-gluon vertex, the three- and the four-gluon vertices, the quark-gluon vertex, etc [27]. However, a priori, such a construction involves more than one scales, and further assumptions about their values need be introduced, in order

\footnotetext{
${ }^{1}$ In fact, as has been explained in detail in [26], an effective charge may also be defined from the gaugeinvariant three-gluon vertex [4].
} 
to express the charge as a function of a single variable. As a general rule in all such a constructions one identifies a RG-invariant quantity formed by a judicious combination of the vertex form-factor and the self-energies associated with the fields entering into the vertex. Let us assume, for example, a vertex with three fields, $\Phi_{i}\left(q_{i}\right), i=1,2,3$, entering ( with $q_{1}+q_{2}+q_{3}=0$ ). Denoting the corresponding propagators by $\Delta_{i}\left(q_{i}\right)$, the relevant vertex form-factor by $V\left(q_{1}, q_{2}, q_{3}\right)$, by $Z_{i}$ the corresponding wave-function renormalization constants, and by $Z_{V}$ the vertex renormalization constant, one can renormalize the coupling such that ${ }^{2} Z_{g}=Z_{V}\left(Z_{1} Z_{2} Z_{3}\right)^{-1 / 2}$, from which follows that the combination

$$
\widehat{r}\left(q_{1}, q_{2}, q_{3}\right) \equiv g^{2} V^{2}\left(q_{1}, q_{2}, q_{3}\right) \Delta_{1}\left(q_{1}\right) \Delta_{2}\left(q_{2}\right) \Delta_{3}\left(q_{3}\right)
$$

is a RG-invariant quantity. As mentioned above, the complication with this definition is that $\widehat{r}\left(q_{1}, q_{2}, q_{3}\right)$ is a function of two kinematic variables. Thus, some additional assumption on the preferred kinematic configuration is usually introduced, such as, for example, $q_{1}^{2}=q_{2}^{2}=q_{3}^{2}=q^{2}$ (and therefore $q_{1} \cdot q_{2}=q_{1} \cdot q_{3}=q_{2} \cdot q_{3}=-q^{2} / 2$ ), which fully specifies the kinematic of the renormalization point.

For the case of the ghost-gluon vertex, let us define in general the following renormalization constants

$$
\begin{aligned}
\Delta\left(q^{2}, \mu^{2}\right) & =Z_{A}^{-1}\left(\mu^{2}\right) \Delta_{0}\left(q^{2}\right), \\
F\left(q^{2}, \mu^{2}\right) & =Z_{c}^{-1}\left(\mu^{2}\right) F_{0}\left(q^{2}\right), \\
\Gamma^{\nu}\left(k, q, \mu^{2}\right) & =Z_{1}\left(\mu^{2}\right) \Gamma_{0}^{\nu}(k, q), \\
g_{0} & =Z_{g^{\prime}}\left(\mu^{2}\right) g^{\prime} .
\end{aligned}
$$

Notice that a priori $Z_{g^{\prime}}$ defined as $Z_{g^{\prime}}=Z_{1} Z_{A}^{-1 / 2} Z_{c}^{-1}$, does not have to coincide with the $Z_{g}$ introduced in (2.10); however, as we will see in the next section, they do coincide by virtue of the basic identity we will derive there.

In the Landau gauge, the form factor $B_{1}$ of Eq. (2.3) is UV finite at one-loop, and therefore, no infinite renormalization constant needs to be introduced at that order; of course, $B_{2}$ must be UV finite in all gauges, and to all orders, otherwise the theory would be non-renormalizable. In order to obtain information about the UV behavior of $B_{1}$ beyond

\footnotetext{
${ }^{2}$ In the MOM prescription, for instance, $Z_{g}$ is determined by requiring that the renormalized vertex at the subtraction point assumes its tree-level value.
} 
one-loop, one usually invokes the non-renormalization theorem of Taylor, which states that for vanishing ghost momentum (see Fig. 3), one has that $B_{1}(-q, q)+B_{2}(-q, q)=1$, to all orders in perturbation theory. Given that $B_{2}$ is finite to all orders (for any kinematic configuration), it follows that $B_{1}(-q, q)$ is also finite to all orders.

In particular, for the Taylor (vanishing incoming ghost momentum) kinematics, $Z_{1}$ will be determined as above explained by demanding that the relevant form factor be equal to its tree-level value after renormalization ${ }^{3}$, i.e., $Z_{1}\left[\left(B_{1}(-q, q)+B_{2}(-q, q)\right]=1\right.$. Then, one will have that

$$
Z_{1}=Z_{g^{\prime}} Z_{A}^{1 / 2} Z_{c}=1
$$

from which follows that

$$
Z_{g^{\prime}}^{-1}=Z_{A}^{1 / 2} Z_{c}
$$

Thus, the product

$$
\widehat{r}\left(q^{2}\right)=g^{\prime 2} \Delta\left(q^{2} ; \mu^{2}\right) F^{2}\left(q^{2} ; \mu^{2}\right)=g_{0} \Delta_{0}\left(q^{2}\right) F_{0}^{2}\left(q^{2}\right),
$$

forms either a dimensionful $\mu$-independent combination or a UV cut-off independent one. Provided that we renormalize the propagators in the MOM scheme with Taylor kinematics (named as "Taylor scheme" in [13]), $\widehat{r}\left(q^{2}\right)$ is a RG-invariant combination.

Therefore, for asymptotically large $q^{2}$, in analogy to Eq. (2.13) one can define an alternative QCD running coupling as

$$
\widehat{r}\left(q^{2}\right)=\frac{\bar{g}_{\mathrm{gh}}^{2}\left(q^{2}\right)}{q^{2}} .
$$

Notice that $\bar{g}_{g h}\left(q^{2}\right)$ has been shown to display the same behavior at any loop order as the ghost-gluon coupling for the Taylor kinematics (see Fig. [3) in [13].

Using then Eq. (2.18), and the fact that

$$
D^{-1}\left(q^{2}\right)=q^{2}\left[1+\frac{9}{4} \frac{C_{\mathrm{A}} g^{2}}{48 \pi} \ln \left(\frac{q^{2}}{\mu^{2}}\right)\right],
$$

it is straightforward to verify that $\bar{g}_{\mathrm{gh}}\left(q^{2}\right)$ and $\bar{g}\left(q^{2}\right)$ displays the same one-loop behavior, since, perturbatively the function $1+G\left(q^{2}\right)$ is the inverse of the ghost dressing function $F\left(q^{2}\right)$. As we will see in the next section, this is nothing more than the one-loop manifestation of the more general identity relating $G\left(q^{2}\right)$ and $F\left(q^{2}\right)$.

3 Recall that the form factor emerging at the Taylor kinematic limit $k_{\mu} \rightarrow-q_{\mu}$ is $B_{1}+B_{2}$. 

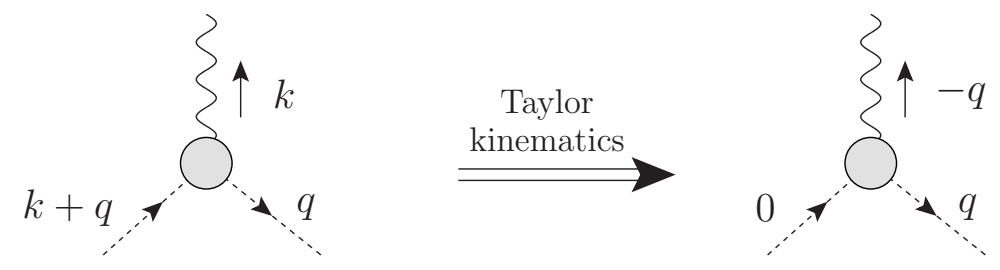

FIG. 3: The ghost-gluon vertex and the Taylor kinematics.

\section{Effective charges from massive gluon propagators}

It is clear from the above analysis that before actually defining the effective charge with either method one constructs two dimensionful RG-invariant quantities, given in Eq. (2.19) and Eq. (2.24), with mass dimension -2. These two quantities share an important common ingredient, namely the scalar cofactor of the gluon propagator, $\Delta\left(q^{2}\right)$, which actually sets the scale. The next step is to extract a dimensionless quantity, that would correspond to the non-perturbative effective charge. Perturbatively, i.e., for asymptotically large momenta, it is clear that the mass scale is saturated simply by $q^{2}$, the bare gluon propagator, and the effective charge is defined by pulling a $q^{-2}$ out of the corresponding RG-invariant quantity ${ }^{4}$.

Of course, as has been firmly established by now, in the IR the gluon propagator becomes effectively massive; therefore, particular care is needed in deciding exactly what combination of mass-scales ought to be pulled out. The correct procedure in such a case has been explained long time ago in the pioneering work of Cornwall [1], and has been applied in various occasions [28]: a "massive" propagator, of the form $\left[q^{2}+m^{2}\left(q^{2}\right)\right]^{-1}$ must be pulled out, where $m^{2}\left(q^{2}\right)$ is a dynamical (i.e., momentum-dependent) $\operatorname{mass}^{5}$.

Before applying this (correct) prescription to the two RG-invariant quantities in question, it is interesting to compare the situation with the more familiar, and conceptually more straightforward, case of the electroweak sector, where the corresponding gauge bosons $(W$ and $Z$ ) are also massive, albeit it through an entirely different mass generation mechanism. Specifically, while the $W$ and $Z$ bosons become massive at tree-level, through the

\footnotetext{
${ }^{4}$ This is equivalent to the standard MOM prescription for the coupling definition.

5 Within the MOM philosophy one may implement the correct prescription by imposing $\Delta^{-1}\left(\mu^{2}\right)=\mu^{2}+m^{2}\left(\mu^{2}\right)$ as the (non-perturbative) MOM renormalization condition for the gluon propagator. This prescription is equivalent to the standard one in the UV, while in the IR it introduces to the anomalous dimensions genuine non-perturbative (Borel non-analytical) terms of the type $\exp \left(-1 / g_{R}\left(q^{2}\right)\right)$, which vanish as $q^{2} \rightarrow \infty$.
} 
standard Higgs mechanism (i.e., fundamental scalars developing a vev), the gluons acquire their (momentum-dependent) masses non-perturbatively, through the dynamical realization of the well-known Schwinger mechanism [29]. Despite the difference in their origin, the masses act in a very similar fashion at the level of the RG-invariant quantity associated with the corresponding gauge boson.

Thus, in the case of the $W$-boson, the corresponding quantity would read (Euclidean momenta)

$$
\widehat{d}_{W}\left(q^{2}\right)=\frac{\bar{g}_{W}^{2}\left(q^{2}\right)}{q^{2}+M_{W}^{2}}
$$

with

$$
\bar{g}_{W}^{2}\left(q^{2}\right)=g_{W}^{2}(\mu)\left[1+b_{W} g_{W}^{2}(\mu) \int_{0}^{1} d x \ln \left(\frac{q^{2} x(1-x)+M_{W}^{2}}{\mu^{2}}\right)-\ldots\right]^{-1}
$$

where $b_{W}=11 / 24 \pi^{2}$, and the ellipses denote the contributions of the fermion families. Clearly, $\widehat{d}_{W}(0)=\bar{g}_{W}^{2}(0) / M_{W}^{2}$, with $\bar{g}_{W}^{2}(0)=g_{W}^{2}(\mu)\left[1+b_{W} g_{W}^{2}(\mu) \ln \left(M_{W}^{2} / \mu^{2}\right)\right]^{-1}$. Evidently, in the deep IR, the coupling freezes at a constant value; Fermi's constant is in fact determined as $4 \sqrt{2} G_{F}=\bar{g}_{W}^{2}(0) / M_{W}^{2}$. Note that in the case of QCD the corresponding combination, $\bar{g}^{2}(0) / m^{2}(0)$ would be similar to a Nambu-Jona-Lasinio type of coupling [30]: at energies below the gluon mass $m$, the "tree-level" amplitude of four-quarks starts looking a lot like that of a four-Fermi interaction [31].

This property of the "freezing" of the coupling can be reformulated in terms of what in the language of the effective field theories is referred to as "decoupling" [32]. At energies sufficiently inferior to their masses, the particles appearing in the loops (in this case the gauge bosons) seize to contribute to the "running" of the coupling. Possibly large logarithmic constants, e.g., $\ln \left(M_{W}^{2} / \mu^{2}\right)$, may be reabsorbed in the renormalized value of the coupling. Of course, the "decoupling" as described above should not be misinterpreted to mean that the running coupling vanishes; instead, as already mentioned, it freezes at a constant, nonzero value. In other words: the "decoupling" does not imply that the theory becomes free (non-interacting) in the IR.

This last clarification is not without relevance for the question at hand, namely the definition of a physically meaningful effective charge. In particular, if one wants to extract an effective charge from an IR-finite gluon propagator (obtained from, e.g., SD studies [25] or from lattice simulations [33, 34, 35]), it would certainly be unwise to insist on the perturbative prescription, and simply factor out a $1 / q^{2}$. Even though one is merely redistributing 

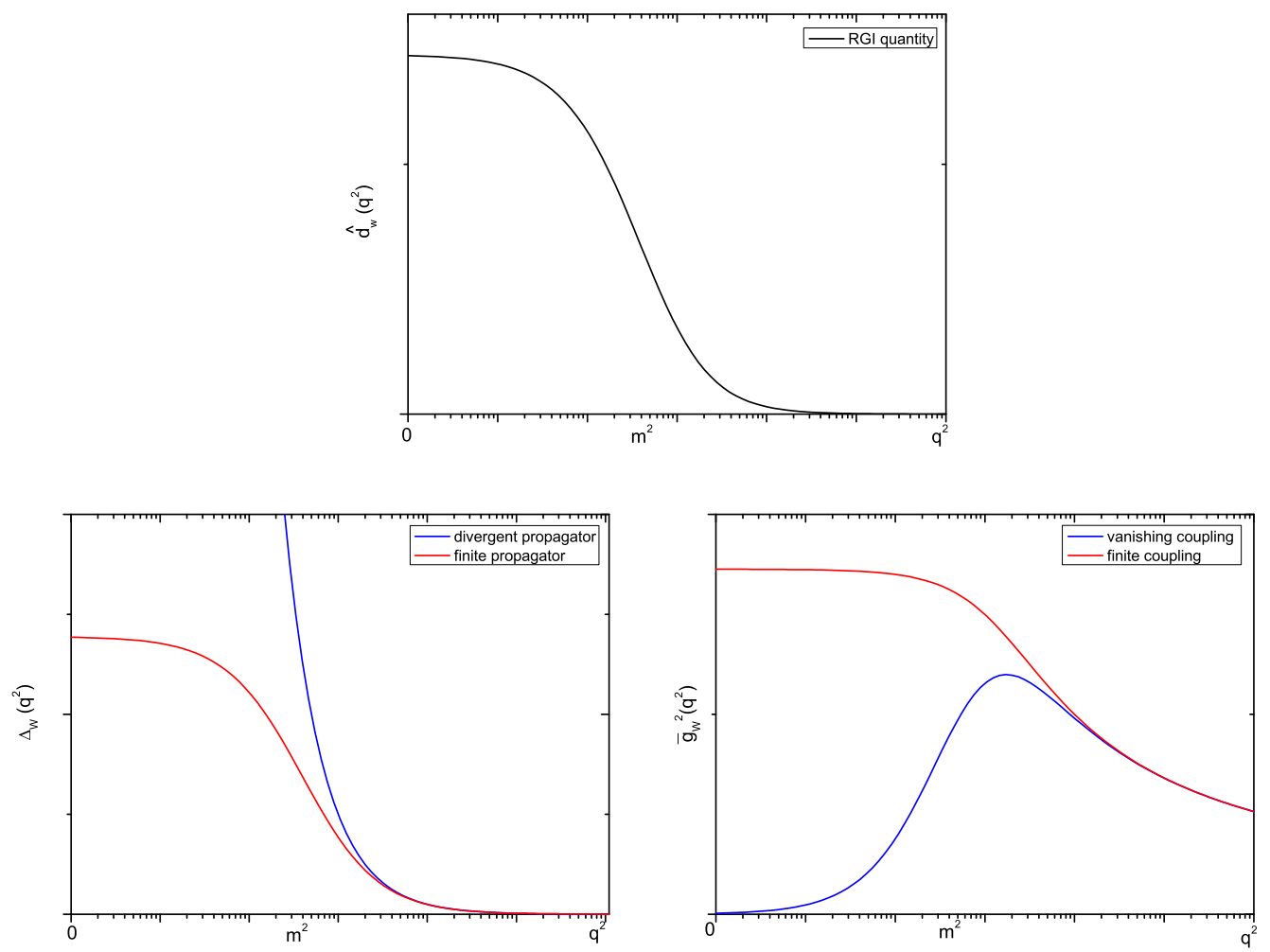

FIG. 4: The same RG-invariant quantity decomposed in two different ways, one giving a divergent propagator and a vanishing coupling, and one giving a finite propagator and a finite coupling

a given function, namely $\widehat{d}_{W}\left(q^{2}\right)$, into two pieces, factoring out $1 / q^{2}$ deprives both of them of any physical meaning. Returning to the electroweak example, the effective coupling so defined would be given by the expression $\widetilde{g}_{W}^{2}\left(q^{2}\right)=q^{2} \widehat{d}_{W}\left(q^{2}\right)$, and so $\widetilde{g}_{W}^{2}(0)=0$; evidently, one would be attempting to describe weak interactions in terms of a massless, IR divergent gauge boson propagator and a vanishing effective coupling (See the curves in blue in Fig. 4). Given that the gluon propagator is finite in the IR, if this latter (wrong) procedure were to be applied to QCD, it would furnish a completely unphysical coupling, namely one that vanishes in the deep IR, where QCD is expected to be (and is) strongly coupled.

As emphasized from the outset, the correct procedure is to factor out of the corresponding RG-invariant combination a "massive" propagator; in the PT case, we write the $\widehat{d}\left(q^{2}\right)$ of 
Eq. (2.19)

$$
\widehat{d}\left(q^{2}\right)=\frac{\bar{g}^{2}\left(q^{2}\right)}{q^{2}+m^{2}\left(q^{2}\right)} .
$$

Given that $\widehat{d}\left(q^{2}\right)=g^{2} \widehat{\Delta}\left(q^{2}\right)$, substituting Eq. (2.29) into (2.15) we obtain

$$
\alpha_{\mathrm{PT}}\left(q^{2}\right)=\left[q^{2}+m^{2}\left(q^{2}\right)\right] \frac{\alpha\left(\mu^{2}\right) \Delta\left(q^{2}\right)}{\left[1+G\left(q^{2}\right)\right]^{2}},
$$

where we have used $\alpha_{\mathrm{PT}}\left(q^{2}\right)=\bar{g}^{2}\left(q^{2}\right) / 4 \pi$. As already mentioned, the dynamical mass $m^{2}\left(q^{2}\right)$ appearing in the definition of $\alpha\left(q^{2}\right)$ is itself running; the explicit form of this running will be discussed in Section IV. Similarly, from the RG-invariant quantity defined starting from the ghost-gluon vertex, given in Eq. (2.24), we have that

$$
\alpha_{\text {gh }}\left(q^{2}\right)=\alpha^{\prime}\left(\mu^{2}\right)\left(q^{2}+m^{2}\left(q^{2}\right)\right) \Delta\left(q^{2}\right) F^{2}\left(q^{2}\right),
$$

where $\alpha^{\prime}\left(\mu^{2}\right)=\bar{g}_{\mathrm{gh}}\left(\mu^{2}\right) / 4 \pi$.

Since $\Delta(0), F(0), G(0)$, and $m(0) \equiv m_{0}$ are all finite (non-vanishing), in the deep IR both couplings assume finite values given by

$$
\begin{aligned}
& \alpha_{\mathrm{PT}}(0)=m_{0}^{2} \alpha\left(\mu^{2}\right) \Delta(0) F^{2}(0), \\
& \alpha_{\mathrm{gh}}(0)=m_{0}^{2} \alpha^{\prime}\left(\mu^{2}\right) \Delta(0)[1+G(0)]^{-2} .
\end{aligned}
$$

\section{DERIVATION OF THE IDENTITY FROM THE DYNAMICAL EQUATIONS}

In this section, we derive the central identity, valid only in the Landau gauge, relating the ghost dressing function with a particular combination of the form-factors $G\left(q^{2}\right)$ and $L\left(q^{2}\right)$ appearing in the tensorial decomposition of $\Lambda_{\mu \nu}$ in Eq. (2.4). The proof hinges crucially on working in the Landau gauge $(\xi=0)$, where the entire gluon propagator $\Delta_{\mu \nu}(k)$ [and not just its self-energy $\left.\Pi_{\mu \nu}(k)\right]$ is transverse, i.e., $k^{\mu} \Delta_{\mu \nu}(k)=0$. As we will see shortly, the operational consequence of this last property is that one can write $q^{\mu} \Delta_{\mu \nu}(k)=(q+$ $k)^{\mu} \Delta_{\mu \nu}(k)$, thus generating for free the appropriate ghost-gluon vertex, as needed.

\section{A. Deriving the relation}

The central relation is obtained as follows. First, consider the standard SD equation for the ghost propagator (Fig [5),

$$
i D^{-1}\left(q^{2}\right)=q^{2}+i g^{2} C_{\mathrm{A}} \int_{k} \Gamma^{\mu} \Delta_{\mu \nu}(k) \Gamma^{\nu}(k, q) D(q+k) .
$$




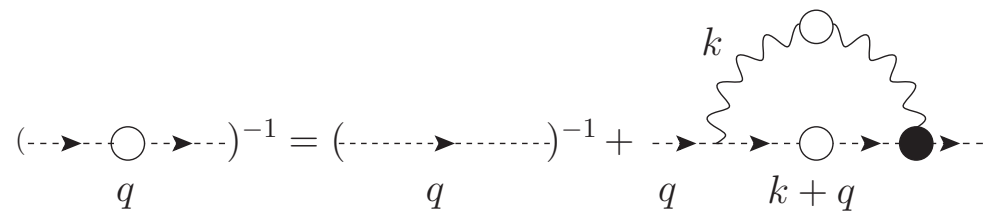

FIG. 5: The SDE for the ghost.

Then, contract both sides of the defining equation (2.4) by the combination $q^{\mu} q^{\nu}$ to get

$$
\left[G\left(q^{2}\right)+L\left(q^{2}\right)\right] q^{2}=g^{2} C_{\mathrm{A}} \int_{k} q_{\rho} \Delta^{\rho \sigma}(k) q^{\nu} H_{\sigma \nu}(k, q) D(k+q) .
$$

Using Eq. (2.5) and the transversality of the full gluon propagator, we can see that the rhs of Eq. (3.2) is precisely the integral appearing in the ghost SDE (3.1). Therefore

$$
\left[G\left(q^{2}\right)+L\left(q^{2}\right)\right] q^{2}=i D^{-1}\left(q^{2}\right)-q^{2}
$$

or, in terms of the ghost dressing function $F\left(q^{2}\right)$ [viz. Eq. (2.2)]

$$
1+G\left(q^{2}\right)+L\left(q^{2}\right)=F^{-1}\left(q^{2}\right) .
$$

The relation of Eq. (3.4), derived here from the SDEs of the theory, has been first obtained in [21], in the framework of the Batalin-Vilkovisky quantization formalism. As was shown there, the relation is a direct consequence of the fundamental BRST symmetry.

Let us study the functions $G\left(q^{2}\right)$ and $L\left(q^{2}\right)$ more closely. From Eq. (2.4) we have that (in $d$ dimensions)

$$
G\left(q^{2}\right)=\frac{1}{(d-1) q^{2}}\left(q^{2} \Lambda_{\mu}^{\mu}-q^{\mu} q^{\nu} \Lambda_{\mu \nu}\right), \quad L\left(q^{2}\right)=\frac{1}{(d-1) q^{2}}\left(d q^{\mu} q^{\nu} \Lambda_{\mu \nu}-q^{2} \Lambda_{\mu}^{\mu}\right),
$$

which then gives, in terms of the SDE integrals

$$
\begin{aligned}
G\left(q^{2}\right) & =\frac{g^{2} C_{\mathrm{A}}}{d-1}\left[\int_{k} \Delta^{\rho \sigma}(k) H_{\sigma \rho}(k, q) D(k+q)+i \frac{1}{q^{2}} \int_{k} q^{\rho} \Delta_{\rho \sigma}(k) \boldsymbol{\Gamma}^{\sigma}(k, q) D(k+q)\right], \\
L\left(q^{2}\right) & =-\frac{g^{2} C_{\mathrm{A}}}{d-1}\left[i \frac{d}{q^{2}} \int_{k} q^{\rho} \Delta_{\rho \sigma}(k) \boldsymbol{\Gamma}^{\sigma}(k, q) D(k+q)+\int_{k} \Delta^{\rho \sigma}(k) H_{\sigma \rho}(k, q) D(k+q)\right] .
\end{aligned}
$$

Inserting the decomposition of Eq. (2.3) and Eq. (2.6) into Eq. (3.6), and setting

$$
f(k, q) \equiv \frac{(k \cdot q)^{2}}{k^{2} q^{2}},
$$


we obtain

$$
\begin{aligned}
G\left(q^{2}\right) & =\frac{g^{2} C_{\mathrm{A}}}{d-1} \int_{k}\left\{(d-1) A_{1}(k, q)-[1-f(k, q)]\left[B_{1}(k, q)-q^{2} A_{2}(k, q)\right]\right\} \Delta(k) D(k+q), \\
L\left(q^{2}\right) & =\frac{g^{2} C_{\mathrm{A}}}{d-1} \int_{k}\left\{(1-d) A_{1}(k, q)+[1-f(k, q)]\left[d B_{1}(k, q)-q^{2} A_{2}(k, q)\right]\right\} \Delta(k) D(k+q),
\end{aligned}
$$

while from Eq. (3.1)

$$
F^{-1}\left(q^{2}\right)=1+g^{2} C_{\mathrm{A}} \int_{k}[1-f(k, q)] B_{1}(k, q) \Delta(k) D(k+q) .
$$

Clearly, Eq. (3.4) is automatically satisfied.

\section{B. Renormalization}

Of course, all quantities appearing in Eq. (3.8) and Eq. (3.9) are unrenormalized (we have suppressed the corresponding subscript "0" for simplicity); in particular, Eq. (3.4) involves unrenormalized $G\left(q^{2}\right), L\left(q^{2}\right)$, and $F\left(q^{2}\right)$. It is easy to recognize, for example, by substituting in the corresponding integrals tree-level expressions, that $F^{-1}\left(q^{2}\right)$ and $G\left(q^{2}\right)$ have the same leading dependence on the UV cutoff $\Lambda_{\mathrm{UV}}$, namely

$$
F_{\mathrm{UV}}^{-1}\left(q^{2}\right)=G_{\mathrm{UV}}\left(q^{2}\right)=\frac{3 g^{2} C_{\mathrm{A}}}{64 \pi^{2}} \ln \left(\frac{\Lambda_{\mathrm{UV}}^{2}}{q^{2}}\right),
$$

while $L\left(q^{2}\right)$ is finite (independent of $\Lambda_{\mathrm{UV}}$ ) at leading order. The next step is therefore to carry out the necessary renormalization.

As already mentioned above, the origin of the basic relation of Eq. (3.4) is the BRST symmetry of the theory; in that sense, Eq. (3.4) has the same origin as the Slavnov-Taylor identities of the theory. Therefore, just as happens with the Slavnov-Taylor identities, Eq. (3.4) should not be deformed after renormalization. Of course, the prototype example of such a situation are the Ward identities of QED; the requirement that the fundamental Ward identity $q^{\mu} \Gamma_{\mu}=S^{-1}(p+q)-S^{-1}(p)$ should retain the same form before and after renormalization leads to the well-known textbook relation $Z_{1}=Z_{2}$ between the corresponding renormalization constants [23]. Similarly, for the case at hand, the renormalization must be carried out in such a way as to preserve the form Eq. (3.4). Specifically, using the defi-

nition given in Eq. (2.16), in order to preserve the relation (3.4) after renormalization, we 
must impose that

$$
Z_{\Lambda}=Z_{c}
$$

In addition, by virtue of (2.5), and for the same reason explained above, we have that, in the Landau gauge $\boldsymbol{\Gamma}_{\nu}(k, q)$ and $H_{\sigma \nu}(k, q)$ must be renormalized by the same renormalization constant, namely $Z_{1}$ [viz. Eq. (2.21)]; for the Taylor kinematics, we have that $Z_{1}=1$ [see Eq. (2.22)].

Then, it is straightforward to renormalize Eq. (3.8) and Eq. (3.9); using

$$
\begin{aligned}
F^{-1}\left(q^{2}, \mu^{2}\right) & =Z_{c}\left(\Lambda_{\mathrm{UV}}^{2}, \mu^{2}\right) F_{0}^{-1}\left(q^{2}, \Lambda_{\mathrm{UV}}^{2}\right), \\
1+G\left(q^{2}, \mu^{2}\right) & =Z_{c}\left(\Lambda_{\mathrm{UV}}^{2}, \mu^{2}\right)\left[1+G_{0}\left(q^{2}, \Lambda_{\mathrm{UV}}^{2}\right)\right], \\
L\left(q^{2}, \mu^{2}\right) & =Z_{c}\left(\Lambda_{\mathrm{UV}}^{2}, \mu^{2}\right) L_{0}\left(q^{2}, \Lambda_{\mathrm{UV}}^{2}\right),
\end{aligned}
$$

we have that

$$
F^{-1}\left(q^{2}\right)=Z_{c}+g^{2} C_{\mathrm{A}} \int_{k}[1-f(k, q)] B_{1}(k, q) \Delta(k) D(k+q),
$$

and

$1+G\left(q^{2}\right)=Z_{c}+\frac{g^{2} C_{\mathrm{A}}}{d-1} \int_{k}\left\{(d-1) A_{1}(k, q)-[1-f(k, q)]\left[B_{1}(k, q)-q^{2} A_{2}(k, q)\right]\right\} \Delta(k) D(k+q)$,

while the equation for $L\left(q^{2}\right)$ remains unchanged, i.e., one simply replaces in the second equation of (3.8) the unrenormalized quantities by renormalized ones. This is consistent with the general observation made in [21], according to which $L\left(q^{2}\right)$ does need its own counterterm, i.e., one proportional to $q_{\mu} q_{\nu}$, in order to get renormalized. The situation is similar to what happens with the $\sigma_{\mu \nu} q^{\nu}$ part of the standard QED vertex: The renormalizability of the theory forbids of course a counterterm proportional to such a tensorial structure; the magnetic form factor (usually denoted by $F_{2}\left(q^{2}\right)$ ) is made finite (beyond one loop) after multiplication by the renormalization constant $Z_{1}$ (whose counterterms are proportional to $\gamma_{\mu}$ ). Thus, while the one-loop answer for $F_{2}$ is finite, at higher orders one gets divergences proportional to $\sigma_{\mu \nu} q^{\nu}$ which are, however, canceled exactly (order by order) by the inclusion of the $Z_{1}$ counterterms in the Feynman graphs of the previous order. For this reason, just as $F_{2}$, despite its one-loop finiteness $L$ depends in general on the UV cutoff $\Lambda_{\mathrm{UV}}^{2}$, as indicated explicitly in Eq. (3.12). 


\section{Calculations and approximations}

In order to study the relevant equations further, we will approximate the form factors $A_{1}(k, q)$ and $B_{1}(k, q)$ with their tree-level values, i.e., $A_{1}(k, q)=B_{1}(k, q)=1$, and $A_{2}(k, q)=0$; according to lattice studies [36], this appears to be a very good approximation. Then, we obtain from Eqs. (3.8) and (3.9)

$$
\begin{aligned}
F^{-1}\left(q^{2}\right) & =Z_{c}+g^{2} C_{\mathrm{A}} \int_{k}[1-f(k, q)] \Delta(k) D(k+q), \\
1+G\left(q^{2}\right) & =Z_{c}+\frac{g^{2} C_{\mathrm{A}}}{d-1} \int_{k}[(d-2)+f(k, q)] \Delta(k) D(k+q), \\
L\left(q^{2}\right) & =\frac{g^{2} C_{\mathrm{A}}}{d-1} \int_{k}[1-d f(k, q)] \Delta(k) D(k+q) .
\end{aligned}
$$

Now, it turns out that if $F$ and $\Delta$ are both IR finite, then

$$
\left.\int_{k}[1-d f(k, q)] \Delta(k) D(k+q)\right|_{q \rightarrow 0}=0,
$$

To see this, one may use the result $\int_{k} k_{\mu} k_{\nu} F(k) \Delta(k)=g_{\mu \nu} d^{-1} \int_{k} F(k) \Delta(k)$, or, equivalently, go to spherical coordinates and use that ${ }^{6}$

$$
\int_{0}^{\pi} d \theta \sin ^{d} \theta\left(1-d \cos ^{2} \theta\right)=0 .
$$

Thus, from Eq. (3.15) we obtain the important result

$$
L(0)=0,
$$

under the assumption that $F$ and $\Delta$ are IR finite. In addition, using (3.16), we obtain

$$
F^{-1}(0)=1+G(0)=Z_{c}+\frac{g^{2} C_{\mathrm{A}}(d-1)}{d} \int_{k} \Delta(k) D(k) .
$$

Note that perturbatively, at one loop, Eq. (3.16) does not hold, because in that case $\Delta(k)$ is not IR finite; consequently, at one loop $L(0) \neq 0$. Specifically in this case, using dimensional regularization, we obtain the $q$-independent result

$$
\int_{k} \frac{1-d f(k, q)}{k^{2}(k+q)^{2}}=-\frac{3}{2} \frac{i}{16 \pi^{2}},
$$

6 Recall that $\int_{0}^{\pi} d \theta \sin ^{n} \theta=\frac{\Gamma\left(\frac{n+1}{2}\right) \Gamma\left(\frac{1}{2}\right)}{\Gamma\left(\frac{n+2}{2}\right)}$. 
which gives

$$
L_{0}\left(q^{2}\right)=\frac{g^{2} C_{\mathrm{A}}}{32 \pi^{2}}
$$

If instead we were to use an IR finite gluon propagator, modeled simply by $\Delta^{-1}(k)=k^{2}-m^{2}$, the same calculation would show that $L_{m}\left(q^{2}\right)$ depends non-trivially on $q^{2}$ [see Eq. (3.26) below], and in fact, $L_{m}(0)=0$.

We next go to the Euclidean space, by setting $-q^{2}=q_{\mathrm{E}}^{2}$, and defining $\Delta_{\mathrm{E}}\left(q_{\mathrm{E}}^{2}\right)=-\Delta\left(-q_{\mathrm{E}}^{2}\right)$, $D_{\mathrm{E}}\left(q_{\mathrm{E}}^{2}\right)=-D\left(-q_{\mathrm{E}}^{2}\right)$, and for the integration measure $\int_{k}=i \int_{k_{\mathrm{E}}}$. Then, using Eq. (2.2) and suppressing the subscript "E", we obtain from Eqs. (3.8) and (3.9)

$$
\begin{aligned}
F^{-1}\left(q^{2}\right) & =Z_{c}-g^{2} C_{\mathrm{A}} \int_{k}[1-f(k, q)] \Delta(k) D(k+q), \\
1+G\left(q^{2}\right) & =Z_{c}-\frac{g^{2} C_{\mathrm{A}}}{d-1} \int_{k}[(d-2)+f(k, q)] \Delta(k) D(k+q), \\
L\left(q^{2}\right) & =-\frac{g^{2} C_{\mathrm{A}}}{d-1} \int_{k}[1-d f(k, q)] \Delta(k) D(k+q) .
\end{aligned}
$$

Next let us introduce spherical coordinates. Setting $q^{2}=x, k^{2}=y$, we have that $k \cdot q=\sqrt{x y} \cos \theta$, and so $(k \cdot q)^{2} / q^{2}=y \cos ^{2} \theta$, and $(k+q)^{2}=x+y+2 \sqrt{x y} \cos \theta$. Moreover, at $d=4$, the measure is given by

$$
\int d^{4} k=2 \pi \int_{0}^{\pi} d \theta \sin ^{2} \theta \int_{0}^{\infty} d y y
$$

Let us first consider the case in which the ghost propagator assumes its tree-level form, namely $D(k+q)=1 /(k+q)^{2}$. Then, using the results

$$
\begin{aligned}
& \int_{0}^{\pi} d \theta \frac{\sin ^{2} \theta}{x+y+2 \sqrt{x y} \cos \theta}=\frac{\pi}{2}\left[\frac{1}{x} \Theta(x-y)+\frac{1}{y} \Theta(y-x)\right] \\
& \int_{0}^{\pi} d \theta \frac{\sin ^{2} \theta \cos ^{2} \theta}{x+y+2 \sqrt{x y} \cos \theta}=\frac{\pi}{8}\left[\frac{1}{x}\left(1+\frac{y}{x}\right) \Theta(x-y)+\frac{1}{y}\left(1+\frac{x}{y}\right) \Theta(y-x)\right],
\end{aligned}
$$

where $\Theta(x)$ is the Heaviside step function, one obtains

$$
\begin{aligned}
1+G(x) & =Z_{c}-\frac{\alpha_{s} C_{\mathrm{A}}}{16 \pi}\left[\frac{1}{x} \int_{0}^{x} d y y\left(3+\frac{y}{3 x}\right) \Delta(y)+\int_{x}^{\infty} d y\left(3+\frac{x}{3 y}\right) \Delta(y)\right], \\
L(x) & =\frac{\alpha_{s} C_{\mathrm{A}}}{12 \pi}\left[\frac{1}{x^{2}} \int_{0}^{x} d y y^{2} \Delta(y)+x \int_{x}^{\infty} d y \frac{\Delta(y)}{y}\right], \\
F^{-1}(x) & =Z_{c}-\frac{\alpha_{s} C_{\mathrm{A}}}{16 \pi}\left[\frac{1}{x} \int_{0}^{x} d y y\left(3-\frac{y}{x}\right) \Delta(y)+\int_{x}^{\infty} d y\left(3-\frac{x}{y}\right) \Delta(y)\right] .
\end{aligned}
$$

Substituting into the equation for $L(x)$ the tree-level value for $\Delta(y)$ we obtain the constant result $L_{0}(x)$ of Eq. (3.21). On the other hand, using $\Delta(y)=\left(y+m^{2}\right)^{-1}$, we find

$$
L_{m}(x)=\frac{\alpha_{s} C_{\mathrm{A}}}{12 \pi}\left\{\frac{1}{x^{2}}\left[\frac{x^{2}}{2}-m^{2} x+m^{4} \ln \left(1+\frac{x}{m^{2}}\right)\right]+\frac{x}{m^{2}} \ln \left(1+\frac{m^{2}}{x}\right)\right\},
$$


from which we clearly see that $L_{m}(0)=0$. In addition, for large $x, L_{m}(x)$ goes over to the massless limit of Eq. (3.21).

The general case for an arbitrary ghost dressing function $F(k+q)$, can be treated by means of the angular approximation. Specifically, one write approximately

$$
\begin{aligned}
1+G(x) & =Z_{c}-\frac{\alpha_{s} C_{\mathrm{A}}}{16 \pi}\left[\frac{F(x)}{x} \int_{0}^{x} d y y\left(3+\frac{y}{3 x}\right) \Delta(y)+\int_{x}^{\infty} d y\left(3+\frac{x}{3 y}\right) \Delta(y) F(y)\right], \\
L(x) & =\frac{\alpha_{s} C_{\mathrm{A}}}{12 \pi}\left[\frac{F(x)}{x^{2}} \int_{0}^{x} d y y^{2} \Delta(y)+x \int_{x}^{\infty} d y \frac{\Delta(y) F(y)}{y}\right] \\
F^{-1}(x) & =Z_{c}-\frac{\alpha_{s} C_{\mathrm{A}}}{16 \pi}\left[\frac{F(x)}{x} \int_{0}^{x} d y y\left(3-\frac{y}{x}\right) \Delta(y)+\int_{x}^{\infty} d y\left(3-\frac{x}{y}\right) \Delta(y) F(y)\right]
\end{aligned}
$$

It is then easy to see (e.g., by means of the change of variables $y=z x$ ) that if $\Delta$ and $F$ are IR finite, then $L(0)=0$, as claimed before. Let us now assume that the renormalization condition for $F(x)$ was chosen to be $F\left(\mu^{2}\right)=1$. This condition, when inserted into the third equation of (3.27), allows one to express $Z_{c}$ as

$$
Z_{c}=1+\frac{\alpha_{s} C_{\mathrm{A}}}{16 \pi}\left[\frac{1}{\mu^{2}} \int_{0}^{\mu^{2}} d y y\left(3-\frac{y}{\mu^{2}}\right) \Delta(y)+\int_{\mu^{2}}^{\infty} d y\left(3-\frac{\mu^{2}}{y}\right) \Delta(y) F(y)\right],
$$

and may be used to cast (3.27) into a manifestly renormalized form. Note that if one choses $F\left(\mu^{2}\right)=1$ then one cannot choose simultaneously $G\left(\mu^{2}\right)=0$, because that would violate the identity of Eq. (3.4), given that $L\left(\mu^{2}\right) \neq 0$. In fact, once $F\left(\mu^{2}\right)=1$ has been imposed, the value of $G\left(\mu^{2}\right)$ is completely determined from its own equation, i.e., the first equation in (3.27).

In addition in the MOM scheme the conventional and PT propagator cannot be made equal at the renormalization point, since the identity (2.15) implies $\widehat{\Delta}^{(-1)}\left(\mu^{2}\right)=\mu^{2}\left[1+G^{2}\left(\mu^{2}\right)\right]^{2}$.

\section{Implications for the effective charges}

After this general discussion, let us now return to the couplings, and discuss the implications of the identity and the dynamics we have derived.

First of all, comparing Eq. (2.12) and Eq. (2.24), it is clear that $g(\mu)=g^{\prime}(\mu)$, by virtue of Eq. (3.11). Therefore, using Eq. (2.15), one can get a relation between the two RG-invariant quantities, $\widehat{r}\left(q^{2}\right)$ and $\widehat{d}\left(q^{2}\right)$, namely

$$
\widehat{r}\left(q^{2}\right)=\left[1+G\left(q^{2}\right)\right]^{2} F^{2}\left(q^{2}\right) \widehat{d}\left(q^{2}\right) .
$$


From this last equality follows that $\alpha_{\mathrm{PT}}$ and $\alpha_{\mathrm{gh}}\left(q^{2}\right)$ are related by

$$
\alpha_{\mathrm{gh}}\left(q^{2}\right)=\left[1+G\left(q^{2}\right)\right]^{2} F^{2}\left(q^{2}\right) \alpha_{\mathrm{PT}}\left(q^{2}\right)
$$

After using Eq. (3.4), we have that

$$
\alpha_{\text {gh }}\left(q^{2}\right)=\left[\frac{1+G\left(q^{2}\right)}{1+G\left(q^{2}\right)+L\left(q^{2}\right)}\right]^{2} \alpha_{\mathrm{PT}}\left(q^{2}\right) .
$$

or, equivalently,

$$
\alpha_{\mathrm{PT}}\left(q^{2}\right)=\alpha_{\mathrm{gh}}\left(q^{2}\right)\left[1+\frac{L\left(q^{2}\right)}{1+G\left(q^{2}\right)}\right]^{2} .
$$

Evidently, the two couplings can only coincide at two points: (i) at $q^{2}=0$, where, due to the fact that $L(0)=0[$ see Eq. (3.18) $]$, we have that

$$
\alpha_{\mathrm{gh}}(0)=\alpha_{\mathrm{PT}}(0)
$$

and (ii) at $q^{2}=\infty$, given that in the deep UV $L\left(q^{2}\right)$ approaches a constant. Note in fact that the two effective charges cannot coincide at the renormalization point $\mu$, where

$$
\alpha_{\mathrm{gh}}\left(\mu^{2}\right)=\left[1-L\left(\mu^{2}\right)\right]^{2} \alpha_{\mathrm{PT}}\left(\mu^{2}\right)
$$

this can be understood also in terms of the discussion following Eq. (3.28).

As we will see in the next section, the numerical analysis reveals that $L\left(q^{2}\right)$ is fairly small compared to $G\left(q^{2}\right)$; thus, even in the region of intermediate momenta, where the difference reaches its maximum, the relative difference between the two charges is less than $5 \%$.

\section{NUMERICAL ANALYSIS}

In this section we will compute the QCD effective charges defined above, using as input for the various Green's functions appearing in their definitions the non-perturbative solutions of the corresponding SDEs, in the Landau gauge. In particular, we will solve numerically a system of three coupled non-linear integral equations, containing $\Delta\left(q^{2}\right), F\left(q^{2}\right)$, and $G\left(q^{2}\right)$ as unknown quantities. Once solutions for these three functions have been obtained, then $L\left(q^{2}\right)$ is fully determined by its corresponding equation, namely the second one in Eq. (3.27). 

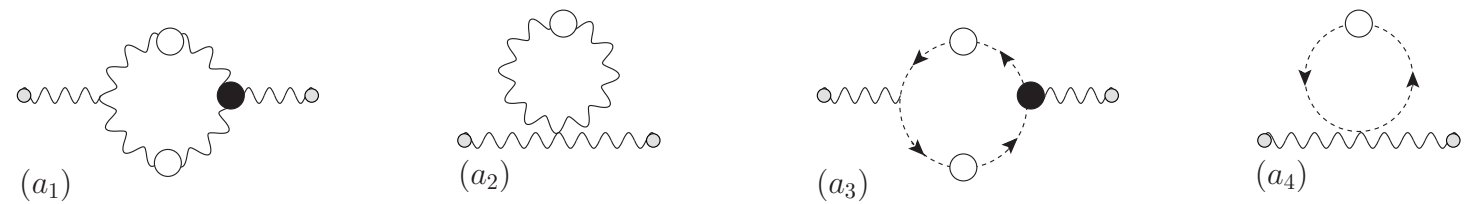

FIG. 6: The new SDE for the gluon propagator at the one-loop dressed level.

\section{A. The system of SD equations}

The two SDEs determining $F\left(q^{2}\right)$ and $G\left(q^{2}\right)$ are given in Eq. (3.27). The SD equation governing $\Delta\left(q^{2}\right)$, is given by [25]

$$
\left[1+G\left(q^{2}\right)\right]^{2} \Delta^{-1}\left(q^{2}\right) P_{\mu \nu}(q)=q^{2} P_{\mu \nu}(q)+i \sum_{i=1}^{4}\left(a_{i}\right)_{\mu \nu}
$$

where the diagrams $\left(a_{i}\right)_{\mu \nu}$ are shown in Fig. 6. As explained in [25], due to the abelian Ward-identities satisfied by the fully-dressed vertices in the PT-BFM scheme, we have that $q^{\mu}\left[\left(a_{1}\right)_{\mu \nu}+\left(a_{2}\right)_{\mu \nu}\right]=q^{\mu}\left[\left(a_{3}\right)_{\mu \nu}+\left(a_{4}\right)_{\mu \nu}\right]=0$. This last property enforces the transversality of the gluon self-energy "order-by-order" in the dressed-loop expansion, which is one of the central features of the gauge-invariant Schwinger-Dyson truncation scheme defined within the PT-BFM framework [18].

After introducing appropriate Ansätze for the aforementioned fully-dressed vertices, we finally arrive at the integral equation

$$
\begin{aligned}
{\left[1+G\left(q^{2}\right)\right]^{2} \Delta^{-1}\left(q^{2}\right) } & =q^{2}-\frac{g^{2} C_{A}}{6}\left[\int_{k} \Delta(k) \Delta(k+q) f_{1}+\int_{k} \Delta(k) f_{2}-\frac{1}{2} \int_{k} \frac{q^{2}}{k^{2}(k+q)^{2}}\right] \\
& +g^{2} C_{A}\left[\frac{4}{3} \int_{k}\left[k^{2}-\frac{(k \cdot q)^{2}}{q^{2}}\right] D(k) D(k+q)-2 \int_{k} D(k)\right],
\end{aligned}
$$

with

$$
\begin{aligned}
& f_{1}=20 q^{2}+18 k^{2}-6(k+q)^{2}+\frac{\left(q^{2}\right)^{2}}{(k+q)^{2}}-(k \cdot q)^{2}\left[\frac{20}{k^{2}}+\frac{10}{q^{2}}+\frac{q^{2}}{k^{2}(k+q)^{2}}+\frac{2(k+q)^{2}}{q^{2} k^{2}}\right] \\
& f_{2}=-\frac{27}{2}-8 \frac{k^{2}}{(k+q)^{2}}+8 \frac{q^{2}}{(k+q)^{2}}+4 \frac{(k \cdot q)^{2}}{k^{2}(k+q)^{2}}-4 \frac{(k \cdot q)^{2}}{q^{2}(k+q)^{2}}
\end{aligned}
$$

The important point is that, by virtue of the massless composite poles introduced into the SDE through the particular Ansätze employed [1, 5, 37], one obtains an IR finite solution for the gluon propagator, i.e., a solution with $\Delta^{-1}(0)>0$, in complete agreement with a large body of lattice data [33, 34, 35]. As explained in detail in [25], the formal expression 

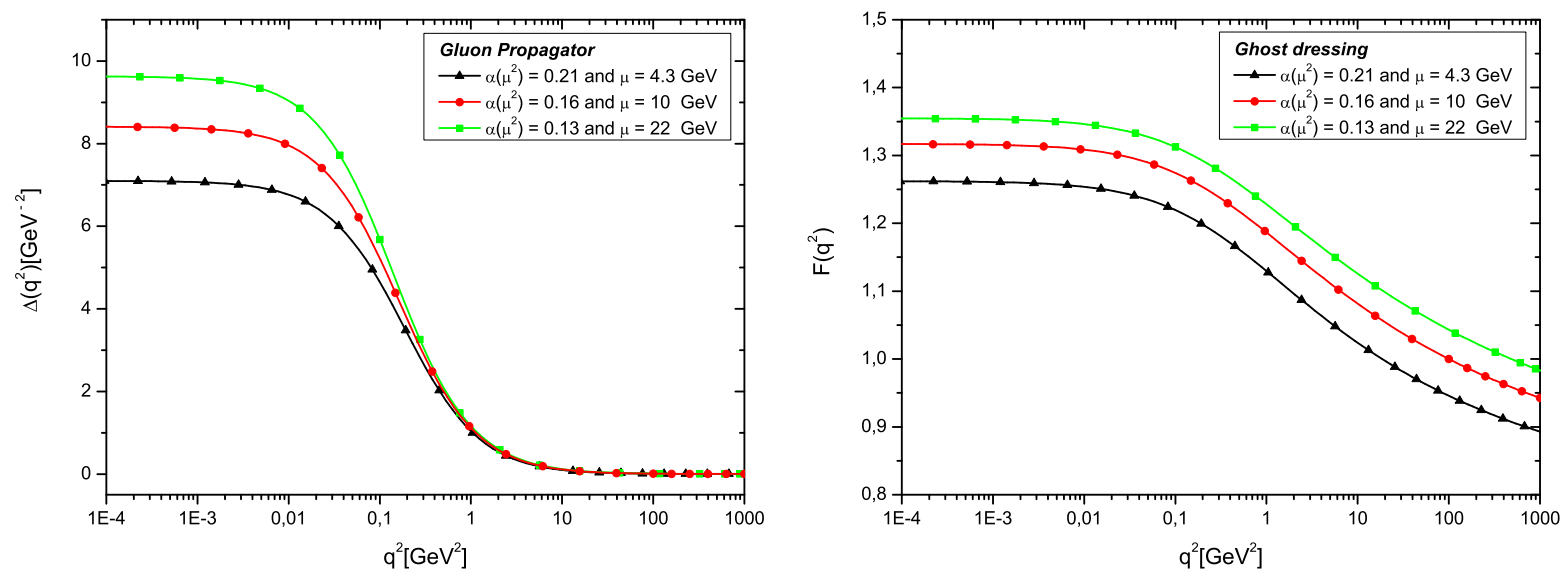

FIG. 7: Left panel: Numerical solutions for the gluon propagator obtained from the SDE using three different renormalization points: $\mu=4.3 \mathrm{GeV}$ and $\alpha\left(\mu^{2}\right)=0.21$ (black curve), $\mu=10 \mathrm{GeV}$ and $\alpha\left(\mu^{2}\right)=0.16$ (red curve), $\mu=22 \mathrm{GeV}$ and $\alpha\left(\mu^{2}\right)=0.13$ (green curve). Right panel: The ghost dressing function $F\left(q^{2}\right)$ obtained from its corresponding SDE and renormalized at the same points.

determining $\Delta^{-1}(0)$ involves quadratically divergent integrals, which may be regulated using the standard rules of dimensional regularization. This procedure leaves the (finite) value of $\Delta^{-1}(0)$ largely undetermined; therefore, in practice, $\Delta^{-1}(0)$ is treated as a free parameter, whose value is to be fixed using phenomenological constraints or lattice data. In addition, and because $\Delta^{-1}(0)$ is finite, the ghost dressing function $F\left(q^{2}\right)$ clearly saturates in the deep IR, reaching a finite value at $q^{2}=0$ (no "enhancement" observed), in agreement with recent lattice data [34, 35], and a variety of independent studies [15, 38].

\section{B. Solutions and checks}

In Fig. 7, we show the numerical results for $\Delta\left(q^{2}\right)$ and $F\left(q^{2}\right)$, renormalized at three different points. On the left panel, the black curve represents the numerical solution of $\Delta\left(q^{2}\right)$ when $\alpha\left(\mu^{2}\right)=0.21$ and $\mu=4.3 \mathrm{GeV}$. The red curve is obtained when $\alpha\left(\mu^{2}\right)=0.16$ and $\mu=10 \mathrm{GeV}$, while for the green curve we used $\alpha\left(\mu^{2}\right)=0.13$ and $\mu=22 \mathrm{GeV}$. On the right panel we plot the corresponding $F\left(q^{2}\right)$ renormalized at the same points.

In Fig. 8 we show the numerical results for the functions $1+G\left(q^{2}\right)$ and $L\left(q^{2}\right)$, using the same renormalization points used previously. The color pattern is also the same as before. For values of $q^{2}<0.1 \mathrm{GeV}^{2}$, we then see that $\left[1+G\left(q^{2}\right)\right]^{2}$ develops a plateau and 

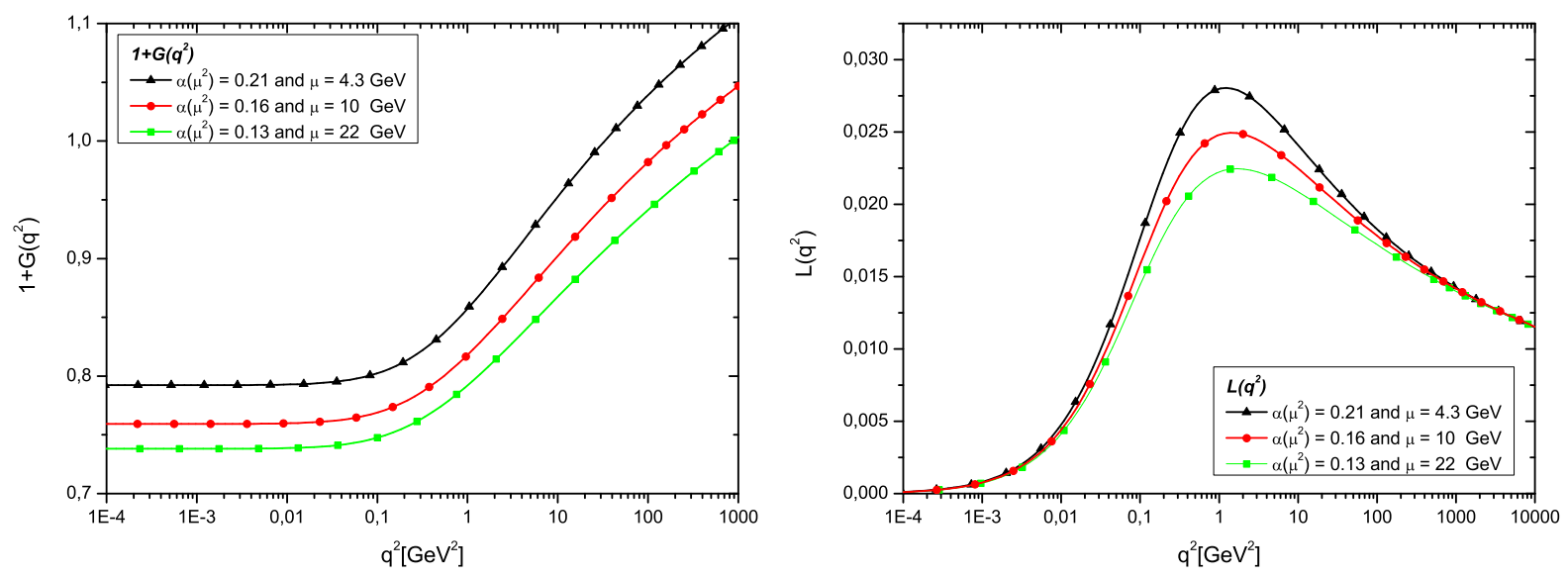

FIG. 8: Left panel: $1+G\left(q^{2}\right)$ determined from Eq. (3.27), using the solutions for $\Delta\left(q^{2}\right)$ and $D\left(q^{2}\right)$ presented in the Fig. 7 at the same renormalization point. Right panel: The function $L\left(q^{2}\right)$ obtained from Eq. (3.27).

saturates at a finite value in the deep IR region. In the UV region, we instead recover the perturbative behavior (2.18). On the other hand, $L\left(q^{2}\right)$ (right panel) shows a maximum in the intermediate momentum region, while, as expected, $L(0)=0$.

With all ingredients defined, the first thing one can check is whether Eq. (2.19) gives rise

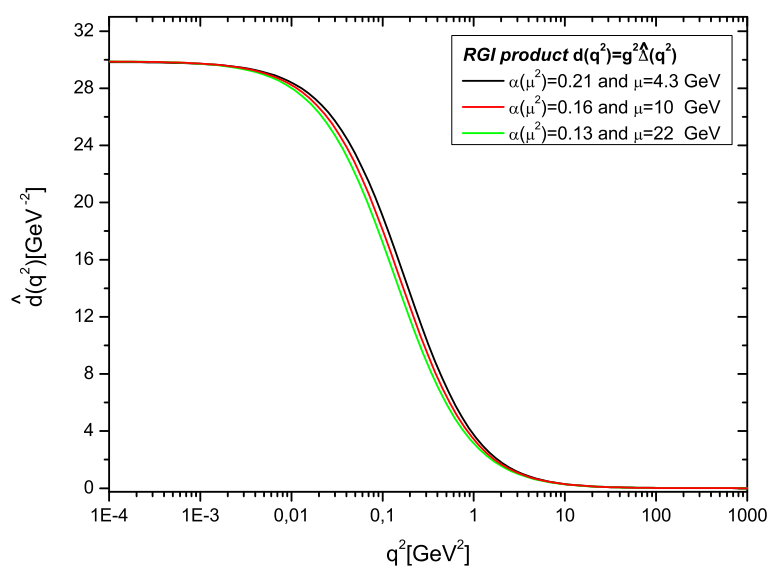

FIG. 9: The product $\widehat{d}\left(q^{2}\right)$ obtained combining the results for $\Delta\left(q^{2}\right)$ and $\left[1+G\left(q^{2}\right)\right]^{2}$ according to Eq. (2.19). 


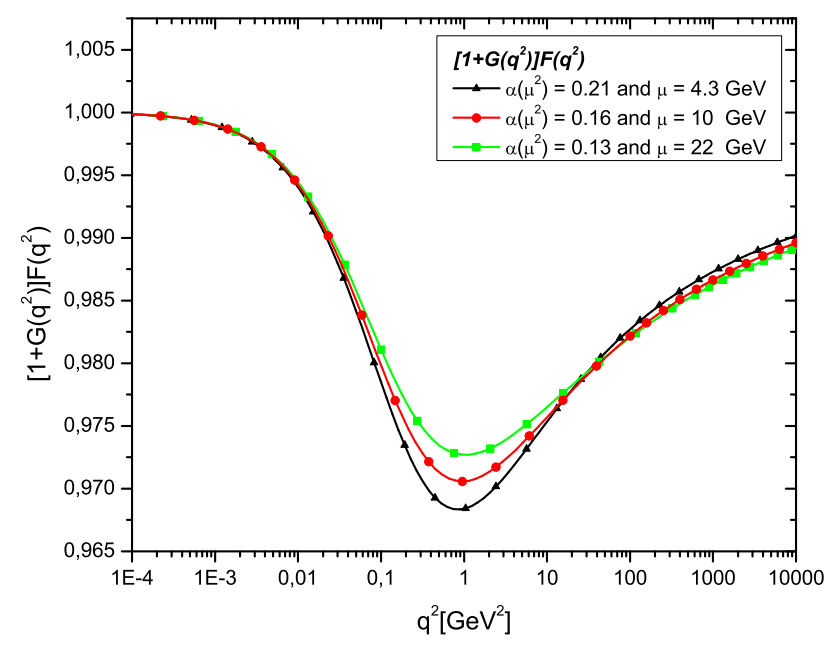

FIG. 10: The product $(1+G) F$ for different values of the renormalization point $\mu$. Note the fine scale of the $y$-axis.

to a RG-invariant combination, as expected. Using the latter definition, we can combine the different data sets for $\Delta\left(q^{2}\right)$ and $\left[1+G\left(q^{2}\right)\right]^{2}$ at different renormalization points, to arrive at the curves shown in Fig. 9. Indeed, we see that the combination $\widehat{d}\left(q^{2}\right)$ is practically independent of the renormalization point chosen.

In addition, from the available solutions we can compute the product $(1+G) F$, which, according to Eq. (3.30), relates the two effective charges of interest. Evidently, since both effective charges are supposed to be RG-invariant quantities, so should be the product $(1+G) F$ relating them. In Fig. 10 we plot $(1+G) F$ for different values of the renormalization point $\mu$; clearly the dependence on $\mu$ is very mild. The theoretical origin of this residual $\mu$-dependence can be traced back to the approximations used for the ghost-gluon vertex $\boldsymbol{\Gamma}_{\nu}$ and the function $H_{\mu \nu}$ (see beginning of subsection $\mathrm{C}$ ). This approximation distorts the multiplicative renormalizability of the corresponding SDEs; indeed, for multiplicative renormalizability to be enforced, one must assume the exact renormalization properties for $\boldsymbol{\Gamma}_{\nu}$ and $H_{\mu \nu}$, as was done in subsection B, where the renormalization was carried out formally. Instead, the approximation employed causes a mismatch in higher orders, which introduces the observed mild dependence on $\mu$. This dependence can be eliminated by resorting to the systematic improvement of the corresponding Ansatz used for $\boldsymbol{\Gamma}_{\nu}$, in the spirit of the prototype QED 


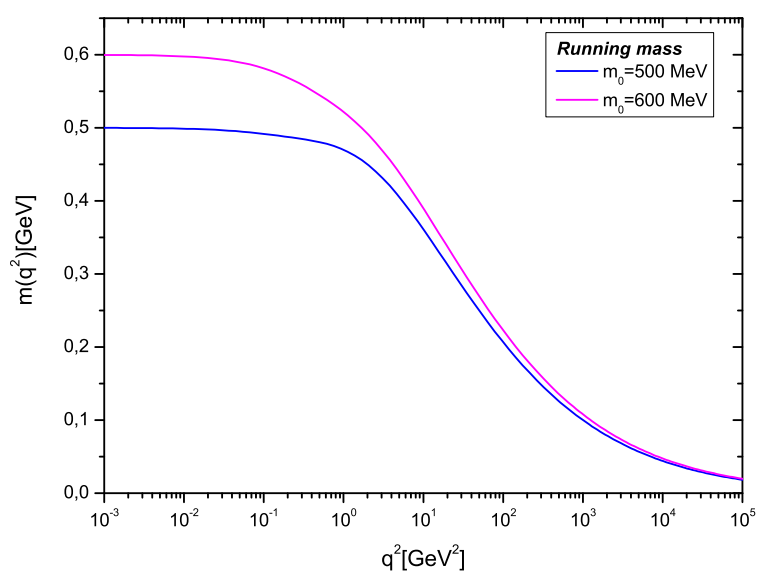

FIG. 11: The behavior of the running mass given by Eq. (4.4) when $m_{0}=500 \mathrm{MeV}$ (blue line) and $m_{0}=600 \mathrm{MeV}$ (magenta line). In both cases we used $\Lambda_{\mathrm{QCD}}=300 \mathrm{MeV}$.

calculations presented in [39], and more recently in [40].

\section{The effective charges}

We can next proceed to extract the non-perturbative running charge $\alpha_{\mathrm{PT}}\left(q^{2}\right)$, defined in Eq. (2.30), by multiplying the results obtained for $\widehat{d}\left(q^{2}\right)$ by the factor $\left[q^{2}+m^{2}\left(q^{2}\right)\right]$. To this end, we will assume that $m^{2}\left(q^{2}\right)$ has a power-law type of running, given by [41, 42]

$$
m^{2}\left(q^{2}\right)=\frac{m_{0}^{4}}{q^{2}+m_{0}^{2}}\left[\ln \left(\frac{q^{2}+2 m_{0}^{2}}{\Lambda_{\mathrm{QCD}}^{2}}\right) / \ln \left(\frac{2 m_{0}^{2}}{\Lambda_{\mathrm{QCD}}^{2}}\right)\right]^{3} .
$$

Notice that when $q^{2} \rightarrow 0$ one has $m^{2}(0)=m_{0}^{2}$. A variety of theoretical and phenomenological estimates place it in the range $m_{0}=350-700 \mathrm{MeV}$ [1, 3, 33, 43]. In Fig. 11 we plot the behavior of $m^{2}\left(q^{2}\right)$ as given by Eq. (4.4), for the two values $m_{0}=500 \mathrm{MeV}$ and $m_{0}=600 \mathrm{MeV}$, which will be used in the rest of this section.

On the left panel of Fig. 12, we show the results for $\alpha_{\mathrm{PT}}\left(q^{2}\right)$ when $m_{0}=500 \mathrm{MeV}$ in Eq. (4.4). The small discrepancy between the three curves is mainly due to the propagation of the tiny residual $\mu$ dependence displayed by the quantity $\widehat{d}\left(q^{2}\right)$ as shown in Fig. 9 , One clearly sees that the effective coupling $\alpha_{\mathrm{PT}}\left(q^{2}\right)$ freezes out and acquires a finite value in the IR, while in the UV it shows the expected perturbative behavior. For $m_{0}=500 \mathrm{MeV}$, one 

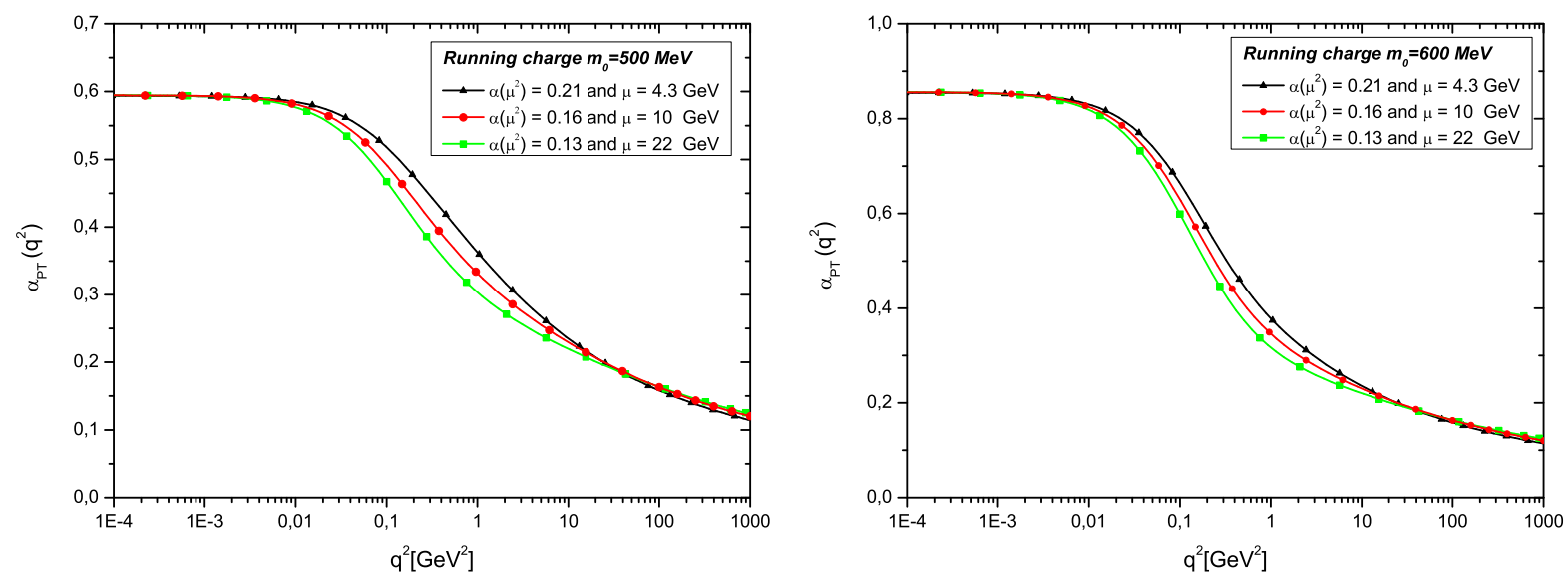

FIG. 12: Left panel: The running charge obtained from (2.30) using the SDE solutions for $\Delta\left(q^{2}\right)$, $D\left(q^{2}\right)$, and $1+G\left(q^{2}\right)$. We use a running mass given by Eq. (4.4) with $m_{0}=500 \mathrm{MeV}$. Right panel: The same for $m_{0}=600 \mathrm{MeV}$.

gets $\alpha_{\mathrm{PT}}(0) \approx 0.6$. One should also notice that the choice of smaller values of $m_{0}$ would not produce a monotonically decreasing $\alpha_{\mathrm{PT}}\left(q^{2}\right)$; instead, one observes the appearance of "bumps" in the IR region. Therefore if one were to introduce the monotonic decrease as an additional requirement of $\alpha_{\mathrm{PT}}\left(q^{2}\right)$, this would provide a lower bound for the possible values of $m_{0}$. Finally, on the right panel of Fig. 12, we show the effective coupling for the case $m_{0}=600 \mathrm{MeV}$. Now, the freezing occurs at the slightly higher value of $\alpha_{\mathrm{PT}}(0) \approx 0.85$. Evidently, the freezing value $\alpha_{\mathrm{PT}}(0)$ increases as one goes to higher values of $m_{0}$.

An accurate fit for the running charges shown in Fig. 12 is provided by the following functional form

$$
\alpha\left(q^{2}\right)=\left[4 \pi b \ln \left(\frac{q^{2}+h\left(q^{2}, m^{2}\left(q^{2}\right)\right)}{\Lambda_{\mathrm{QCD}}^{2}}\right)\right]^{-1},
$$

with the function $h\left(q^{2}, m^{2}\left(q^{2}\right)\right)$ given by

$$
h\left(q^{2}, m^{2}\left(q^{2}\right)\right)=\rho_{1} m^{2}\left(q^{2}\right)+\rho_{2} \frac{m^{4}\left(q^{2}\right)}{q^{2}+m^{2}\left(q^{2}\right)} .
$$

Our best fits to the numerical results for $\alpha_{\mathrm{PT}}\left(q^{2}\right)$ using Eq. (4.5) above are shown in Fig. 13,

Finally, we compare numerically the two effective charges, $\alpha_{\mathrm{PT}}\left(q^{2}\right)$ and $\alpha_{\mathrm{gh}}\left(q^{2}\right)$. The results are shown in Fig. 14, where $\widehat{r}\left(q^{2}\right)$ is compared with $\widehat{d}\left(q^{2}\right)$ (left panel), and $\alpha_{\text {gh }}\left(q^{2}\right)$ with $\alpha_{\mathrm{PT}}\left(q^{2}\right)$ (right panel). As anticipated, the curves coincide in the deep IR and UV, and 

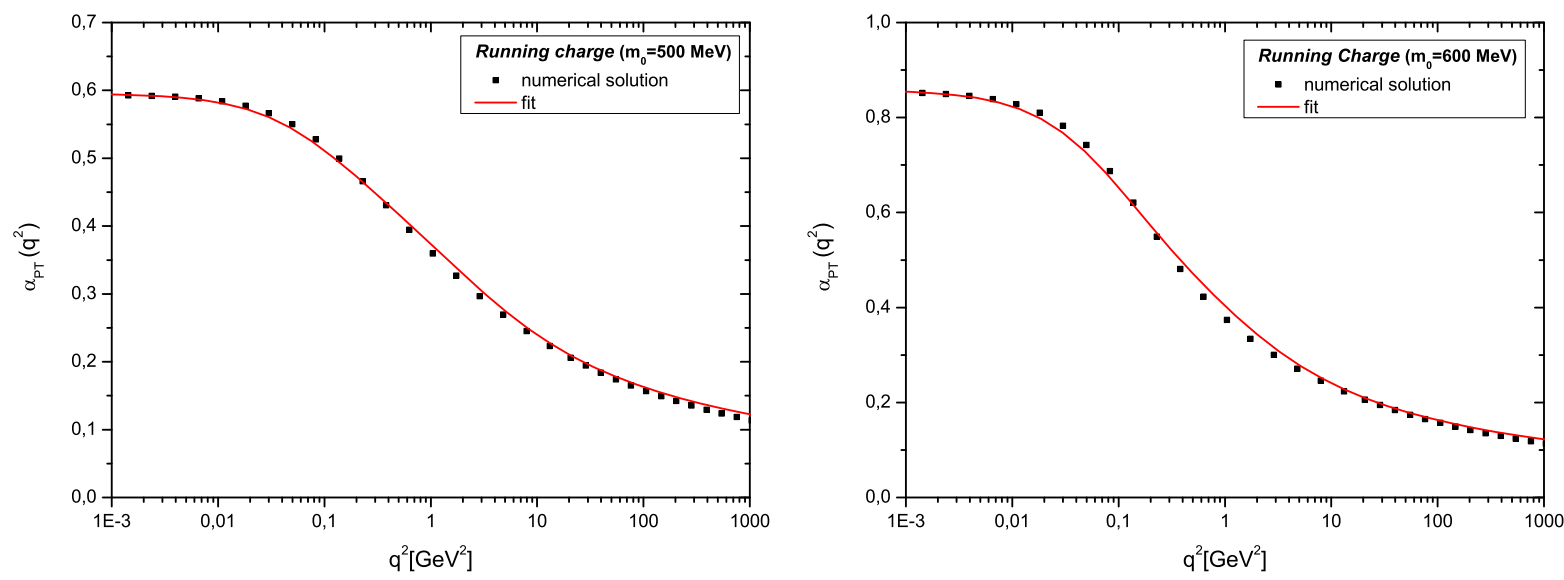

FIG. 13: Left panel: The fit given by Eq. (4.5) for $m_{0}=500 \mathrm{MeV}$; in this case the best fit values correspond to $\rho_{1}=4.5$, and $\rho_{2}=-2$. Right panel: Same as before in the case $m_{0}=600 \mathrm{MeV}$; in this case the best fit parameters are $\rho_{1}=2.2$, and $\rho_{2}=-1.25$.

differ only slightly in the intermediate region. To produce both curves, we have factored out a mass of $m_{0}=500 \mathrm{MeV}$, whose dynamical running is again given in Eq. (4.4); equivalently, one could use directly Eq. (3.30).

\section{CONCLUSIONS}

In this article we have presented a detailed comparison between the two QCD effective charges, $\alpha_{\mathrm{PT}}\left(q^{2}\right)$ and $\alpha_{\mathrm{gh}}\left(q^{2}\right)$, obtained within two vastly different frameworks: the PT (and BFM) on the one hand, and the ghost-gluon vertex (with the Taylor-kinematics) on the other. It turns out that their dynamics involves the gluon propagator $\Delta\left(q^{2}\right)$ (in the Landau gauge) as a common ingredient, entering in both $\alpha_{\mathrm{PT}}\left(q^{2}\right)$ and $\alpha_{\mathrm{gh}}\left(q^{2}\right)$, and two different ingredients, which participate in a non-trivial identity. This identity, which is valid only in the Landau gauge, relates the ghost dressing function, $F\left(q^{2}\right)$, with the two form-factors, $G\left(q^{2}\right)$ and $L\left(q^{2}\right)$, appearing in the Lorentz decomposition of a special Green's function, originating from the ghost sector of the theory.

The two QCD effective charges have been computed using as input the non-perturbative solutions of a system of three coupled non-linear integral equations, first derived in [25], containing $\Delta\left(q^{2}\right), F\left(q^{2}\right)$, and $G\left(q^{2}\right)$ as unknown quantities. The solutions obtained from the 

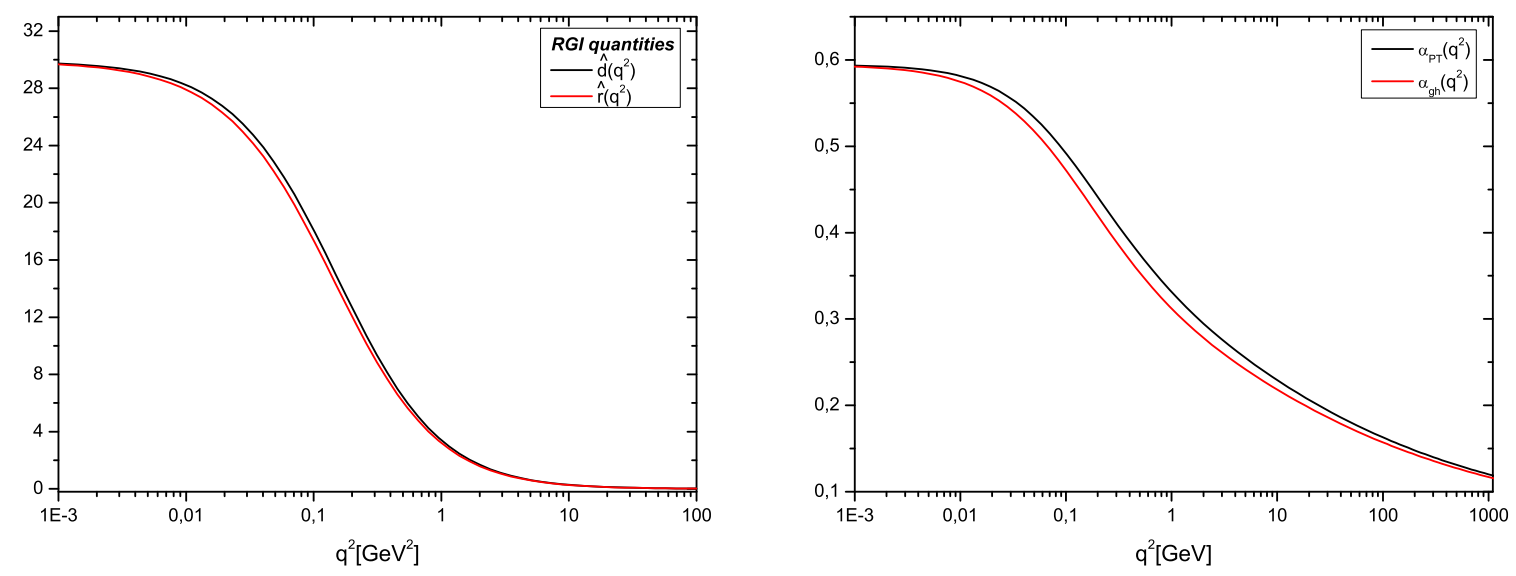

FIG. 14: Left panel: Comparison between the RG-invariant products $r\left(q^{2}\right)$ (red curve) and $\widehat{d}\left(q^{2}\right)$ (black curve). Right panel: Comparison between $\alpha_{\mathrm{gh}}\left(q^{2}\right)$ (red curve) and $\alpha_{\mathrm{PT}}\left(q^{2}\right)$ (black curve) when a mass of $m_{0}=500 \mathrm{MeV}$ is factored out. In both plots the difference between the curves appear in the intermediate regime of momenta being entirely due to the function $L\left(q^{2}\right)$.

above system of SDEs for $\Delta\left(q^{2}\right)$ and $F\left(q^{2}\right)$ - and subsequently fed into the defining equations of the effective charges- are in qualitative agreement with recent results from large-volume lattices, both for $S U(2)$ [34] and $S U(3)$ [35]: both quantities reach finite (non-vanishing) values in the deep IR. One important consequence of the central identity (and the dynamics encoded in the relevant equations) is that the two charges are identical not only in the deep UV, where asymptotic freedom manifests itself, but also in the deep IR, where they "freeze" at the same non-vanishing value.

As already mentioned in section [V] at the level of the SDE for the gluon propagator, namely Eq. (4.2), the value of $\Delta(0)$ is a free parameter. The value chosen for $\Delta(0)$ affects (in a non-linear way) the IR values of the RG-invariant quantities, namely $\widehat{d}(0)$ and $\widehat{r}(0)$, which, in turn, restricts the values of the gluon mass, $m_{0}$, and the freezing value of the effective charges. Throughout the analysis presented in section IV the criterion used for choosing the values of $\Delta(0)$ was that the resulting values for $m_{0}$ and $\alpha_{\mathrm{gh}}(0)$, (or $\left.\alpha_{\mathrm{PT}}(0)\right)$ would be numerically compatible with those obtained from a variety of phenomenological studies [43]. Specifically, values for $m_{0}$ in the range of $350-700 \mathrm{MeV}$ and $\alpha_{\mathrm{PT}}(0) \approx 0.7 \pm 0.3$. Notice, however, a subtle point that may be of relevance when carrying out such comparisons. 
The effective charge assumed in most of the aforementioned studies is that of [1], which has a very particular functional form, and corresponds to the standard PT construction, where the Feynman gauge of the BFM is dynamically singled out. Instead, for the reason explained in subsection 2.2, the present analysis is based on the generalized PT [24], which eventually projects one to the Landau gauge of the BFM. It would be interesting to reach a quantitative understanding of how the aforementioned difference in the gauges affects the phenomenological values of the gluon mass and of the freezing of the effective charge. Calculations in this direction are already in progress.

As has been emphasized in [25], even though the solutions of the SDE system are in qualitative agreement with the aforementioned lattice results, they display a considerable quantitative discrepancy from them. Specifically, $\Delta\left(q^{2}\right)$ differs significantly in the region of intermediate momenta, and the value of the ghost dressing function is about a factor of two less than that obtained on the lattice. These discrepancies, in turn, are expected to affect the numerical values (but not the qualitative features) of quantities computed using them as input. In particular, it should be interesting to obtain the QCD effective charges studied here using as input the lattice results for $\Delta\left(q^{2}\right)$ and $F\left(q^{2}\right)$, and [indirectly, using, e.g., the first equation in (3.27) ] for $G\left(q^{2}\right)$; we hope to address this issue in a future work.

\section{Acknowledgments}

The research of J. P. is supported by the European FEDER and Spanish MICINN under grant FPA2008-02878, and the Fundación General of the UV. The work of J. R-Q. is supported by the Spanish MEC grant FPA-2006-13825.

[1] J. M. Cornwall, Phys. Rev. D 26, 1453 (1982).

[2] A. C. Mattingly and P. M. Stevenson, Phys. Rev. D 49, 437 (1994); Y. L. Dokshitzer, G. Marchesini and B. R. Webber, Nucl. Phys. B 469 (1996) 93; A. M. Badalian and V. L. Morgunov, Phys. Rev. D 60, 116008 (1999); A. C. Aguilar, A. A. Natale and P. S. Rodrigues da Silva, Phys. Rev. Lett. 90, 152001 (2003); S. J. Brodsky, S. Menke, C. Merino and J. Rathsman, Phys. Rev. D 67, 055008 (2003); S. J. Brodsky, Fizika B 13, 91 (2004); M. Baldicchi and G. M. Prosperi, Phys. Rev. D 66, 074008 (2002); G. Grunberg, Phys. Rev. 
D 29, 2315 (1984); Phys. Rev. D 73, 091901 (2006); D. V. Shirkov and I. L. Solovtsov, Phys. Rev. Lett. 79, 1209 (1997); G. M. Prosperi, M. Raciti and C. Simolo, Prog. Part. Nucl. Phys. 58, 387 (2007).

[3] J. M. Cornwall, arXiv:0904.3758 [hep-ph].

[4] J. M. Cornwall and J. Papavassiliou, Phys. Rev. D 40, 3474 (1989).

[5] A. C. Aguilar and J. Papavassiliou, JHEP 0612, 012 (2006).

[6] N. J. Watson, Nucl. Phys. B 494, 388 (1997); D. Binosi and J. Papavassiliou, Nucl. Phys. Proc. Suppl. 121, 281 (2003).

[7] A. Denner, G. Weiglein and S. Dittmaier, Phys. Lett. B 333, 420 (1994); S. Hashimoto, J. Kodaira, Y. Yasui and K. Sasaki, Phys. Rev. D 50, 7066 (1994); J. Papavassiliou, Phys. Rev. D 51, 856 (1995).

[8] D. Binosi and J. Papavassiliou, Phys. Rev. D 66(R), 111901 (2002); J. Phys. G 30, 203 (2004).

[9] L. F. Abbott, Nucl. Phys. B 185, 189 (1981).

[10] J. C. Taylor, Nucl. Phys. B 33, 436 (1971).

[11] W. J. Marciano and H. Pagels, Phys. Rept. 36, 137 (1978).

[12] J. C. R. Bloch, A. Cucchieri, K. Langfeld and T. Mendes, Nucl. Phys. B 687, 76 (2004); O. Oliveira and P. J. Silva, Braz. J. Phys. 37, 201 (2007); A. Sternbeck, K. Maltman, L. von Smekal, A. G. Williams, E. M. Ilgenfritz and M. Muller-Preussker, PoS LAT2007 (2007) 256.

[13] Ph. Boucaud, F. De Soto, J. P. Leroy, A. Le Yaouanc, J. Micheli, O. Pene and J. RodriguezQuintero, Phys. Rev. D 79 (2009) 014508.

[14] L. von Smekal, A. Hauck and R. Alkofer, Annals Phys. 267, 1 (1998) [Erratum-ibid. 269, 182 (1998)]; J. C. R. Bloch, Few Body Syst. 33, 111 (2003).

[15] Ph. Boucaud, Th. Bruntjen, J.P. Leroy, A. Le Yaouanc, A.Y. Lokhov, J. Micheli, O. Pene, J. Rodriguez-Quintero, JHEP 0606 (2006) 001; Ph. Boucaud, J.P. Leroy, A. Le Yaouanc, A.Y. Lokhov, J. Micheli, O. Pene, J. Rodriguez-Quintero, C. Roiesnel, JHEP 0703 (2007) 076; Ph. Boucaud, J. P. Leroy, A. L. Yaouanc, J. Micheli, O. Pene and J. Rodriguez-Quintero, JHEP 0806 (2008) 012; JHEP 0806 (2008) 099.

[16] P. A. Grassi, T. Hurth and M. Steinhauser, Annals Phys. 288, 197 (2001).

[17] D. Binosi and J. Papavassiliou, Phys. Rev. D 66, 025024 (2002).

[18] D. Binosi and J. Papavassiliou, Phys. Rev. D 77(R), 061702 (2008); JHEP 0811, 063 (2008).

[19] T. Kugo, arXiv:hep-th/9511033. 
[20] T. Kugo and I. Ojima, Prog. Theor. Phys. Suppl. 66, 1 (1979).

[21] P. A. Grassi, T. Hurth and A. Quadri, Phys. Rev. D 70, 105014 (2004).

[22] K. I. Kondo, arXiv:0904.4897 [hep-th].

[23] See, for example, C. Itzykson and J-B. Zuber, Quantum Field Theory (McGraw-Hill, New York, 1980).

[24] A. Pilaftsis, Nucl. Phys. B 487, 467 (1997).

[25] A. C. Aguilar, D. Binosi and J. Papavassiliou, Phys. Rev. D 78, 025010 (2008).

[26] M. Binger and S. J. Brodsky, Phys. Rev. D 74, 054016 (2006) arXiv:hep-ph/0602199.

[27] R. Alkofer, C. S. Fischer and F. J. Llanes-Estrada, Phys. Lett. B 611, 279 (2005) [Erratumibid. 670, 460 (2009)].

[28] A. C. Aguilar and J. Papavassiliou, AIP Conf. Proc. 964, 312 (2007); J. Phys. Conf. Ser. 110, 022040 (2008); A. C. Aguilar, D. Binosi and J. Papavassiliou, PoS LC2008, 050 (2008).

[29] J. S. Schwinger, Phys. Rev. 125, 397 (1962); J. S. Schwinger, Phys. Rev. 128, 2425 (1962).

[30] Y. Nambu and G. Jona-Lasinio, Phys. Rev. 122, 345 (1961); Phys. Rev. 124, 246 (1961).

[31] We thank A. Santamaria for pointing this out to us.

[32] T. Appelquist and J. Carazzone, Phys. Rev. D 11, 2856 (1975); S. Weinberg, Phys. Lett. B 91, 51 (1980); G. Rodrigo, A. Pich and A. Santamaria, Phys. Lett. B 424, 367 (1998).

[33] C. W. Bernard, Phys. Lett. B 108, 431 (1982); J. E. Mandula and M. Ogilvie, Phys. Lett. B 185, 127 (1987); C. W. Bernard, C. Parrinello and A. Soni, Phys. Rev. D 49, 1585 (1994); C. Alexandrou, P. de Forcrand and E. Follana, Phys. Rev. D 63, 094504 (2001); F. D. R. Bonnet, P. O. Bowman, D. B. Leinweber and A. G. Williams, Phys. Rev. D 62, 051501 (2000); F. D. R. Bonnet, P. O. Bowman, D. B. Leinweber, A. G. Williams and J. M. Zanotti, Phys. Rev. D 64, 034501 (2001).

[34] A. Cucchieri and T. Mendes, PoS LAT2007, 297 (2007); Phys. Rev. Lett. 100, 241601 (2008); arXiv:0904.4033 [hep-lat].

[35] I. L. Bogolubsky, E. M. Ilgenfritz, M. Muller-Preussker and A. Sternbeck, PoS LAT2007, 290 (2007); Phys. Lett. B 676, 69 (2009).

[36] A. Cucchieri, T. Mendes and A. Mihara, JHEP 0412, 012 (2004); E. M. Ilgenfritz, M. MullerPreussker, A. Sternbeck and A. Schiller, arXiv:hep-lat/0601027.

[37] R. Jackiw and K. Johnson, Phys. Rev. D 8, 2386 (1973); J. M. Cornwall and R. E. Norton, Phys. Rev. D 8 (1973) 3338; E. Eichten and F. Feinberg, Phys. Rev. D 10, 3254 (1974). 
[38] D. Dudal, J. A. Gracey, S. P. Sorella, N. Vandersickel and H. Verschelde, Phys. Rev. D 78, 065047 (2008).

[39] J. E. King, Phys. Rev. D 27, 1821 (1983).

[40] A. Kizilersu and M. R. Pennington, arXiv:0904.3483 [hep-th].

[41] A. C. Aguilar and J. Papavassiliou, Eur. Phys. J. A 35, 189 (2008).

[42] M. Lavelle, Phys. Rev. D 44, 26 (1991).

[43] F. Halzen, G. I. Krein and A. A. Natale, Phys. Rev. D 47, 295 (1993); M. B. Gay Ducati, F. Halzen and A. A. Natale, Phys. Rev. D 48, 2324 (1993); M. Consoli and J. H. Field, Phys. Rev. D 49, 1293 (1994); F. J. Yndurain, Phys. Lett. B 345 (1995) 524; A. Szczepaniak, E. S. Swanson, C. R. Ji and S. R. Cotanch, Phys. Rev. Lett. 76, 2011 (1996); J. H. Field, Phys. Rev. D 66, 013013 (2002); A. C. Aguilar, A. Mihara and A. A. Natale, Phys. Rev. D 65, 054011 (2002); Int. J. Mod. Phys. A 19, 249 (2004); E. G. S. Luna, A. F. Martini, M. J. Menon, A. Mihara and A. A. Natale, Phys. Rev. D 72, 034019 (2005). 